Proceedings of the Prehistoric Society 84, 2018, pp. 359-385 (C) The Prehistoric Society. This is an Open Access article, distributed under the terms of the Creative Commons Attribution licence (http://creativecommons.org/ licenses/by/4.0/), which permits unrestricted reuse, distribution, and reproduction in any medium, provided the original work is properly cited.

doi:10.1017/ppr.2018.11 First published online 05 November 2018

\title{
Bronze Age Textile \& Wool Economy: The Case of the Terramare Site of Montale, Italy
}

\author{
By SERENA SABATINI ${ }^{1}$, TIMOTHY EARLE ${ }^{2}$ and ANDREA CARDARELLI ${ }^{3}$
}

At the onset of the 2nd millennium BC, a wool economy emerged across continental Europe. Archaeological, iconographical, and written sources from the Near East and the Aegean show that a Bronze Age wool economy involved considerable specialised labour and large scale animal husbandry. Resting only on archaeological evidence, detailed knowledge of wool economies in Bronze Age Europe has been limited, but recent investigations at the Terramare site of Montale, in northern Italy, document a high density of spindle whorls that strongly supports the existence of village-level specialised manufacture of yarn. Production does not appear to have been attached to an emerging elite nor was it fully independent of social constraints. We propose that, although probably managed by local elites, wool production was a community-based endeavour oriented towards exports aimed at obtaining locally unavailable raw materials and goods.

Keywords: Bronze Age, Italy, craft production, spindle whorls, community of practice, contexts of specialisation, political economy, commodity flows

Several studies show consistent and important roles for textile production, trade, and consumption, especially of wool items, in the Bronze Age political economies of the eastern Mediterranean (Barber 1991; Burke 2010; Nosch 2011; 2015; Wright 2013; Breniquet \& Michel 2014; Harlow et al. 2014; Andersson Strand \& Nosch 2015a). As analytical techniques have expanded, studies also shed light on textiles and textile production at this time in continental Europe (Bender Jørgensen 1992; Gillis \& Nosch 2007; Gleba 2008; 2012; Gleba \& Mannering 2012; Grömer et al. 2013; Grömer 2016). Although analyses of textile fragments and tools exist, much remains to be done to grasp the socio-cultural and political significance of textiles and, particularly, the wool textile economy of Bronze Age

\footnotetext{
${ }^{1}$ Department of Historical Studies, University of Gothenburg, Box 200, 40530 Göteborg, Sweden, Email: serena.sabatini@archaeology.gu.se

${ }^{2}$ Department of Anthropology, Northwestern University, 1810 Hinman Avenue, Evanston, IL 60208 USA

${ }^{3}$ Faculty of Letters \& Philosophy, Rome University $\mathrm{La}$ Sapienza, Piazzale Aldo Moro, 5, 00185 Roma RM, Italy
}

Europe. We need to study specific and variable contexts of production, trade, and consumption. Because textiles are not normally preserved archaeologically, tools for textile manufacture, especially ceramic or stone spindle whorls, are critical for investigating context and scale of production. In this article we present a study of spindle whorls from the Bronze Age Terramare settlement of Montale in the Po Valley, Italy, and their role in community-based specialised wool economy. An exceptional number of spindle whorls (over 4000 items) have been found at this settlement. What is the significance of this concentration? Our thesis is that such a high density of whorls suggests intense textile production and that at least one of the outcomes is likely to have been the provision of exports for trade against required goods such as metals. The characteristics of the archaeological record from both Montale and the rest of the Terramare area (eg, Bernabò Brea et al. 1997a; Cardarelli 2014; Pacciarelli 2016, 168-70) does not provide clear evidence for significant social inequality either in the settlements (with larger or richer households) or in the 
necropoleis (with distinguished graves). From a sociopolitical point of view, we therefore suppose that even large-scale craft practices, such as intense textile production, might have been the outcome of communitybased engagement that did not result in significant social inequality.

\section{BRONZE AGE TEXTILES AND WOOL IN CONTINENTAL EUROPE}

Any attempt to understand Bronze Age textile production beyond the coasts of the Mediterranean is like doing a jigsaw. Although plentiful, the evidence for textile production is solely archaeological, since no written documents exist. Additionally, the archaeological record is not homogeneously spread, either chronologically or geographically. It seems, therefore, profitable to make use of comparative data and information from areas outside continental Europe, such the Aegean and the Near East.

Although admittedly documenting much more complex political economies than those found in continental Europe, Aegean and Near Eastern written sources provide insight for interpreting broader archaeological patterns. Studies, in particular, of Linear B tablets from palace archives in the Aegean (Killen 2007; 2015, 1-3; Nosch 2011; Del Freo et al. 2010) and of Assyrian letters from the lower town of the Anatolian city of Kaneš/Kültepe (Wisti Lassen 2010; Michel \& Veenhof 2010; Michel 2014) record resources and labour investment throughout chaine opératoires of textile production, and also quality and quantities of demand. Because textiles are seldom preserved (Skals et al. 2015), texts, which are often concerned with wool and woollen products, provide an important, contemporaneous record as to textile manufacture and trade (McCorriston 1997; Michel \& Nosch 2010; Wright 2013, 397-8; Breniquet \& Michel 2014; Harlow et al. 2014; Nosch 2015). All in all, Bronze Age wool production in the Aegean and eastern Mediterranean was a complicated and dynamic enterprise. A growing demand for clothing of different quality fuelled production activities in specific centres that managed collection and redistribution of raw materials and textile making. It was a year-around activity that relied on access to vast numbers of sheep/goats, paid and/or unfree specialised craft-labour, and conspicuous elite consumption (eg, Burke 2010; Breniquet \& Michel 2014). But can this model be applied to Europe more generally?
Archaeological examples of wool fragments from across Europe (Broholm \& Hald 1940; Bender Jørgensen 1992; Bender Jørgensen \& Rast-Eicher 2016; CinBa database; Gleba \& Mannering 2012; Grömer et al. 2013; Rast-Eicher \& Bender Jørgensen 2013) suggest that, early in the 2 nd millennium $\mathrm{BC}$, wool became a sought-after material beyond the Mediterranean coastal region. At about the same time, changes in sheep culling suggest that, in some continental regions, raising sheep became geared to wool production (eg, Vretemark 2010). In addition, strontium isotope analyses of woollen clothing from several 14th century BC oak-log coffin graves (Denmark) document that most preserved textiles from these elite contexts were made with non-local wool (Frei et al. 2015; 2017). Considering that no convincing archaeological evidence exists for textile manufacture in Bronze Age Scandinavia (eg, Bergerbrant 2007, 49; forthcoming; Sofaer et al. 2013, 480), and in disagreement with earlier proposals suggesting that wool might have been a Nordic export (eg, Randsborg 2011, 110), those isotopic analyses hint at the existence of a continental Bronze Age trade providing wool to the north. The archaeological evidence from the Bronze Age Po valley in northern Italy, as presented in this paper, represents a convincing case that, during the 2 nd millennium $\mathrm{BC}$, wool economies emerged and developed beyond the coastal region of the Mediterranean to supply continental demand.

\section{BRONZE AGE WOOL IN CONTINENTAL ITALY}

The earliest spun wool fibres from the Italian peninsula (Bazzanella 2012; Bazzanella \& Mayr 2009, 35, 41-6, 79-8) are from the Early Bronze Age Alpine lake dwellings (Polada Culture, c. $2200-1650$ BC). ${ }^{1}$ Although scanty, they suggest that both the material and the production process were well-known, at least in the northern part of the peninsula, long before the Middle Bronze Age evidence from Montale. The earliest pure woollen fabric is a fragment of tabby weave from the Terramare settlement of Castione dei Marchesi (Parma province), likely dated to the Middle Bronze Age (c. 1650-1300 BC) $)^{2}$ (Bazzanella 2012, 209). Microscopic analyses of its fibres suggest that the wool came from sheep resembling today's Soay breed (Gleba 2012, 328-9), which moult once a year to yield c. $0.3-0.9 \mathrm{~kg}$ of wool (Robson \& Ekarius 2011, 195). This figure corresponds well to the wool unit in Aegean archives, expressed by the sign $* 145 /$ LANA, which seems to signify a wool sack of 
c. $3 \mathrm{~kg}$, containing four adult sheep fleeces of $c .750 \mathrm{~g}$ or ten fleeces of $c .300 \mathrm{~g}$ from mixed flocks (Del Freo et al. 2010, 340-4). It seems, therefore, that local Terramare sheep most likely resembled, at least in terms of yearly wool yield, those of the Aegean; and that archive documents might provide useful reference material.

According to a neo-Sumerian (c. $2050 \mathrm{BC}$ ) source, as many as $4 \mathrm{~kg}$ of a fourth-class wool (valued on a 1 [royal] to 5 [poorest quality] scale) were necessary just to obtain an average $(g u z-z a)$ fabric of $c .3 .5 \times 3.5 \mathrm{~m}$ (eg, Andersson Strand \& Cybulska 2013, 113-8). Considering the probable low productivity, in terms of yearly wool yield, of the Terramare sheep, as in the Aegean and the near East (Halstead 1999; Biga 2011; Firth 2014), any Middle Bronze Age specialised wool production in the Po plain would have required management of large herds. As discussed below, a good number of Terramare sites, including Montale, show evidence of intense sheep husbandry. Although sheep provide a range of other products as well, it is evident that wool was, at least potentially, a widely available raw material.

\section{TERRAMARE AND BRONZE AGE TEXTILE PRODUCTION IN THE PO VALLEY}

To investigate contexts and scale of textile production beyond the coastal zone of the Mediterranean, we consider the Terramare culture and its settlement of Montale (Modena province). Terramare defines Middle/Recent Bronze Age (Fig. 1) populations of the central part of the Po plain in northern Italy (Bernabò Brea et al. 1997a; Blake 2014, 113-49; Cardarelli 2009a; 2014; 2015; Vanzetti 2013). ${ }^{3}$ As an archaeological complex, Terramare displays distinctive settlement organisation and land-use. Initially in the Middle Bronze Age, settlements were primarily small (typically 1-2 ha), with estimated populations of $125 / 130$ inhabitants per ha (Cardarelli 2015, 167) confined within substantial fortifications. Subsequently, from Middle Bronze Age 3 into the Recent Bronze Age 1 (Fig. 1), a form of site hierarchy emerged with some larger settlements over 10 ha that held populations of perhaps 1000 or more (eg, Pacciarelli 2016, 16871). At the same time, extensive irrigation systems have also been documented (eg, Cremaschi et al. 2006, 89; Vanzetti 2013, 271-2). For European prehistory, the Terramare irrigation complexes represent an unusually high investment in engineered landscapes, and have been interpreted as probably being associated with community (corporate) ownership (Cardarelli 2015, 168). Terramare fortified settlements probably asserted a willingness of the community to 'stand its ground' in defence of landscape capital (see Earle 2017) and mobile wealth such as crops, raw materials, textiles, and, to a certain extent, animals (see Cardarelli 2009b). Existence for war-like violence is seen in the necropolis of Olmo di Nogara from the neighbouring Verona Province, north of the Po River, where several skeletons had received dramatic wounds (Canci et al. 2015; Pulcini 2014, 130-43).

In this study, we concentrate on the Terramare settlement of Montale situated in the landscape of the Po plain in the Modena province, which is open and fertile, and in close vicinity to the mountainous areas of the local Apennines (Fig. 2; Bernabò Brea et al. 1997b; Cardarelli 2006; Cavazzuti \& Putzolo 2015). In Roman times this province had a dense human population, intensive agriculture, substantial animal husbandry, favoured among other things by vicinity to the Appennines summer pastures, and was renowned for its wool production (Corti 2012). Archaeozoological evidence suggests that specialised wool production also existed here in the Bronze Age. Compositions of domesticated animals in the Terramare culture broadly (De Grossi Mazzorin 2013) and at the site of Montale specifically (Table 1), show that sheep/goat herding was significant (De Grossi Mazzorin \& Ruggini 2009). Ovicaprids were consistently present on the plain throughout the Middle and Recent Bronze Age, increasing through time to more than $50 \%$ of the animal assemblage at some settlements (De Grossi Mazzorin 2013, table 1). Although the archaeozoological data from many sites, including Montale, have been only published preliminarily, where information about culling strategies are available, it would seem that a mixed pastoral economy, producing both meat and wool, dominated across the plain, while only minor attention was paid to milk production (De Grossi Mazzorin 2013; Riedel 1989; 2004). The presence of clay sheep figurines indicates that they had a social and symbolic significance (Desantis 2011, 38; Bianchi \& Bernabò Brea 2012, fig. 1). ${ }^{4}$

Terramare communities in general appear to have exploited local environmental, technological, and organisational advantages to meet subsistence needs and to produce exports to exchange for needed nonlocal commodities. Metal tools, for example, were widely produced and used, but no local sources of 
THE PREHISTORIC SOCIETY

\begin{tabular}{|c|c|c|c|c|}
\hline Montale & Italy & Greece & $\begin{array}{l}\text { Central } \\
\text { Europe }\end{array}$ & $\begin{array}{l}\text { Northern } \\
\text { Europe }\end{array}$ \\
\hline $\begin{array}{c}\text { Phase } \\
\text { I }\end{array}$ & $\begin{array}{l}\text { Middle Bronze Age } \\
\text { 2A } \\
1550-1500 \text { BC }\end{array}$ & $\begin{array}{l}\text { Late Helladic IIA } \\
\qquad 1600-1500 \mathrm{BC}\end{array}$ & $\begin{array}{l}\text { Bronzezeit B1 } \\
1600-1500 \mathrm{BC}\end{array}$ & $\begin{array}{c}\text { Period IB } \\
1600-1500 \mathrm{BC}\end{array}$ \\
\hline $\begin{array}{c}\text { Phase } \\
\text { II }\end{array}$ & $\begin{array}{c}\text { Middle Bronze Age 2B } \\
\qquad 1500-1450 \text { BC }\end{array}$ & Late Helladic & & \\
\hline $\begin{array}{l}\text { Phases } \\
\text { III-VI }\end{array}$ & $\begin{array}{c}\text { Middle Bronze Age } \\
\text { 3A } \\
1450-1400 \text { BC }\end{array}$ & $\begin{array}{c}\text { IIB-IIIA1 } \\
1500-1400 \mathrm{BC}\end{array}$ & $\begin{array}{c}\text { Bronzezeit } \\
\text { B2-C } \\
1500-1300 \mathrm{BC}\end{array}$ & $\begin{array}{c}\text { Period II } \\
1500-1300 \mathrm{BC}\end{array}$ \\
\hline $\begin{array}{l}\text { Phases } \\
\text { VII-VIII }\end{array}$ & $\begin{array}{c}\text { Middle Bronze Age 3B } \\
1400-1325 / 1300 \text { BC }\end{array}$ & $\begin{array}{c}\text { Late Helladic } \\
\text { IIIA2 } \\
1400-1300 \mathrm{BC}\end{array}$ & & \\
\hline $\begin{array}{l}\text { Phases } \\
\text { IX-XI }\end{array}$ & $\begin{array}{l}\text { Recent Bronze Age } 1 \\
1325 / 1300-1225 / 1200 \\
\text { BC } \\
\text { Recent Bronze Age } 2 \\
1225 / 1200-1150 \text { BC }\end{array}$ & $\begin{array}{l}\text { Late Helladic IIIC } \\
\qquad 1200-1100\end{array}$ & $\begin{array}{l}\text { Bronzezeit D } \\
1300-1200 \mathrm{BC}\end{array}$ & $\begin{array}{c}\text { Period III } \\
1300-1100 \mathrm{BC}\end{array}$ \\
\hline
\end{tabular}

Fig. 1.

Montale's archaeological phases and contemporary main Bronze Age chronologies

metal were available in the plain. For some Terramare communities, a likely export in exchange for the metal might have been textile products. In this respect, the case of Montale, analysed here, might not have been an isolated one. It is, for instance, possible that specialised weaving activity existed at Beneceto (Parma province), where hundreds of fragmentary loom weights have been recovered (Lincetto 2006, 138-56). Also, at Poviglio (Reggio Emilia province), weaving might have been specialised; a conspicuous number of loom weights and probable evidence of standing warp-weighted looms have been recovered in various structures from different parts of that settlement dated to different Bronze Age phases (Bernabò Brea et al. 2003; Bianchi 2004). ${ }^{5}$
Material from 19th century collections, as recorded in Modena Civic Museum registers, provides a striking picture of different frequencies of textile tools from various provincial sites (Table 2). Although these partly unsystematic collections do not offer a safe base for further analyses and comparisons, they provide a good indication of the likely different politico-economic choices of the various settlements as to the intensity of textile manufacture. On the basis of the remarkable quantity of recovered textile tools, Montale provides good evidence for understanding one context of Bronze Age textile production. It suggests, we argue, that it was a community-based specialist production, as defined by Cathy Costin, characterised by 'autonomous individuals or household-based production 


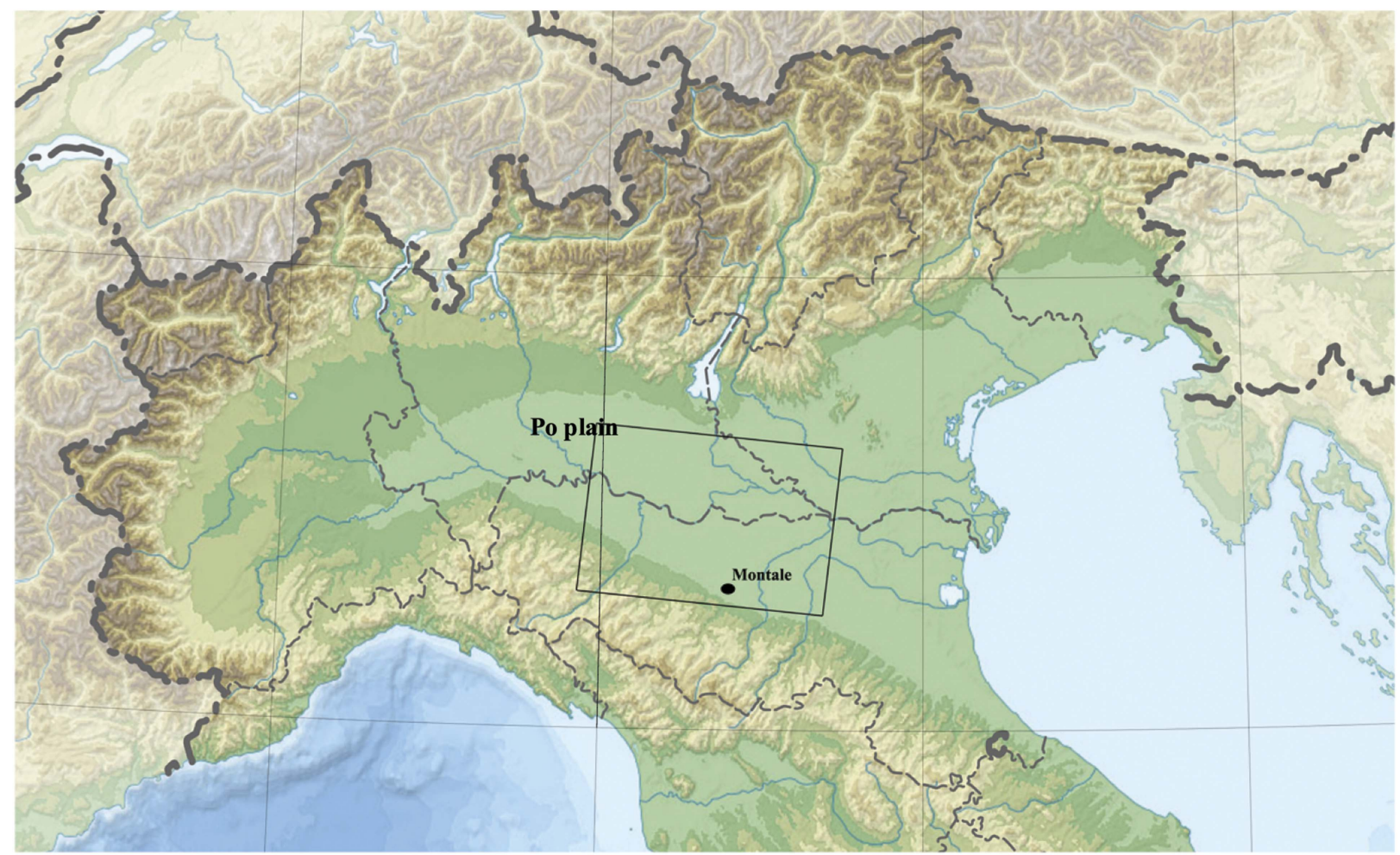

Fig. 2.

The Po plain in Northern Italy with the site of Montale and the area of the terramare

TABLE 1: ANIMAL POPULATION AT MONTALE. APPROXIMATE PERCENTAGE VALUES (STRATIGRAPHIC EXCAVATIONS) (COURTESY OF JACOPO DE GROSSI MAZZORIN)

\begin{tabular}{lccccc}
\hline & Sheep & Goat & $\begin{array}{c}\text { Total sheepl } \\
\text { goat }\end{array}$ & Pig & Cattle \\
\hline MBA2 & 40 & 7.2 & 47.2 & 41.4 & 11.4 \\
MBA3 & 40 & 8 & 49.8 & 37.7 & 12.5 \\
RBA1 & 48 & 13.6 & 61.6 & 28.1 & 10.3 \\
\hline
\end{tabular}

units, aggregated within a single community, producing for unrestricted regional consumption' (Costin 1991, 8).

\section{THE TERRAMARE SETTLEMENT OF MONTALE}

Montale was a typical 1 ha fortified Terramare settlement, which was probably home to a local group of perhaps 130 people. It was surrounded by a massive ditch c. $40 \mathrm{~m}$ wide and $3 \mathrm{~m}$ deep, which would have been filled with water (Fig. 3) to serve several functions including defence (Cardarelli \& Labate 2009a, 28-30). There is no evidence to suggest a social hierarchy at Montale, although some form of local leadership was most probably involved in the construction of both the ditch and the imposing defensive embankment that lay between the ditch and the settlement. The embankment was still preserved for a width of $10 \mathrm{~m}$ and an height of $2 \mathrm{~m}$ at the end of the 19th century (Cardarelli \& Labate 2009a). The site was partly investigated during the 19th century (cf. Fig. 3), but it is only thanks to recent stratigraphic excavations of a $c .45 \mathrm{~m}^{2}$ portion of the settlement (luckily spared by the local manure quarry works, see Fig. 3) that a densely settled space could be revealed. The material from the excavation also helped establish an 11-phase internal chronology from 1600/1550 to $1250 / 1200$ BC (cf. Fig. 1). The results of the excavation show that houses tended to be built and rebuilt within what look like precisely allocated spaces (Cardarelli \& Labate 2009b, fig. 69). They also revealed that the very same space that was occupied by dwellings during Phases I-IV could be used for metallurgical activities during Phase $\mathrm{V}$ and return to accommodate housing during the following Phase VI, though in slightly different positions to the earlier structures. In Phase VIII a granary was also present in the excavated 
area while, during the remaining phases, no structures could be identified (Cardarelli \& Labate 2009b). Phase II is best preserved archaeologically and parts of two different buildings and of the space/street between them, dated to this phase, show that the settlement living quarters were organised in an orthogonal layout, in a fashion similar to that investigated at Poviglio, for instance (Bernabò Brea et al. 2003).

What makes Montale exceptional among Bronze Age settlements, not only in the Po plain but also on a wider continental scale, is its unusually high density of textile tools, particularly spindle whorls (cf. eg, Table 2). Over $90 \%$ of the textile tools from here were collected in the 19th century, when compost for farming was being quarried. At this time, Modena Museum partly supervised the recovery of archaeological material (cf. Fig. 3), comprising thousands of finds, although without contextual information (Cardarelli 2009b, 16-18). Additional archaeological material comes from well-documented, modern excavations (eg, Candelato et al. 2002; Cardarelli 2009b). Finds include items relating to spinning (spindle whorls), weaving (in particular loom weights, possibly also loom combs and at least one loom sword, cf. Cardarelli 2009b, fig. 80.17), and sewing implements (needles). ${ }^{6}$

\section{MONTALE'S SPINDLE WHORLS}

According to the Modena Civic Museum register, 4454 spindle whorls were collected during the 19th century (Table 2), of which 4089 nearly complete whorls are still preserved. At the same time, 127 loom weights, of which 78 are today preserved in the collection, were also brought to the Modena Museum (Sabatini in press). During the recent stratigraphic excavations a further 182 whorls (Tables $3 \& 4$ ) and 17 loom weights were recovered. Considering that many tools $(54 \%$ of the spindle whorls $(\mathrm{N}=98$; Table 3$)$ and $52 \%$ of the loom weights $(\mathrm{N}=9$, cf. Sabatini in press) from recent excavations are fragmentary, the original number of both spindle whorls and loom weights from the Montale quarry excavations must have been much higher than the recorded total of whole textile tools. ${ }^{7}$

This paper focuses on the clay spindle whorls, which are the principal textile tools recovered from Montale. Spindle whorls are flywheels that, fixed on a spindle shaft, help maintain rotation for spinning (Barber 1991, 51-4; Olofsson et al. 2015, 77-8). Spinning is the act of 'twisting and drawing out (or drafting) the fibres of the raw material into a thread' (Barber 1991, 41). Although spinning can be done in many ways (eg, Barber 1991, 39-51; Bender Jørgensen 2012, 129), the 4000+ whorls recovered at Montale and their routine presence in other Terramare sites and throughout Italy from the Neolithic (eg, Gleba 2008, 104), suggests that using clay whorls was the locally preferred technique for spinning thread. Spinning is a time-consuming task (Bender Jørgensen 2012; Olofsson et al. 2015, 84) and, indeed, it dominates labour time in the textile chaine opératoire. Recent tests (Andersson Strand \& Cybulska 2013) confirmed that, of $c .124$ working days needed to produce a $3.5 \times 3.5 \mathrm{~m}$ fabric of average quality from raw wool, as recorded in the neoSumerian text mentioned above (Waetzold 1972, T32), one worker would have had to be occupied for over half the time (c. 67 days) just spinning the necessary warp and weft thread. In addition, experiments demonstrate that the level of required skills and time increase consistently when spinning thin, high quality threads (Bender Jørgensen 2012, 129; Andersson Strand \& Cybulska 2013, 116-8). Although ancient written sources do not seem to document trade in yarn, the production of thread, carried out by carefully recorded specialised labour, must have had a crucial role in both Near Eastern and Aegean economies (see, for instance, Del Freo et al. 2010, 354-6; Firth \& Nosch 2012; Siennika 2014), and we can assume that it was important in contemporary European economies as well.

Montale's whorls are of various types that are typical of the region with some types showing considerable standardisation as to shape and decoration (Fig. 4; Bianchi 2004, fig. 280-1; Leonardi 2012). Only the items from the modern excavations underwent a typological analysis. They have been divided in nine main types: truncated conical (eg, Fig. 4E), biconical asymmetric (eg, Fig. 4C-D), biconical asymmetric with protuberances (eg, Fig. 4F), biconical (eg, Fig. 4B), biconical with concentric marks (eg, Fig. 4A), convex-cylindrical (eg, Fig. 4H), disc-shaped (eg, Fig. 4I), globular (eg, Fig. 4G), and pin-head like (eg, Fig. 4J). Most of the sub-types within each of the main types recur across the sequence (Table 5). Because the stratigraphic excavation were limited (cf. Fig. 3), these samples may be unrepresentative, and so the following hypotheses should be taken with some caution. The greater and more articulated presence of whorls during Phase II might, for instance, depend on the fact 
TABLE 2: FINDS COLLECTED DURING THE 19TH CENTURY FROM TERRAMARE SETTLEMENTS OF THE MODENA PROVINCE, AS RECORDED IN MODENA CIVIC MUSEUM REGISTERS (COURTESY OF GIANLUCA PELLACANI)

\begin{tabular}{|c|c|c|c|c|c|c|c|c|c|}
\hline Site & $\begin{array}{l}\text { Estimated } \\
\text { chronology }\end{array}$ & $\begin{array}{l}\text { Estimated original } \\
\text { size }\end{array}$ & $M a S L$ & $\begin{array}{l}\text { Ceramic } \\
\text { objects }\end{array}$ & $\begin{array}{l}\text { Spindle } \\
\text { whorls }\end{array}$ & $\begin{array}{l}\text { Loom } \\
\text { weights }\end{array}$ & $\begin{array}{c}\text { Total (ceramic } \\
\text { objects + spindle } \\
\text { whorls + loom weights) }\end{array}$ & $\begin{array}{c}\% \text { spindle } \\
\text { whorls of } \\
\text { total }\end{array}$ & $\begin{array}{l}\% \text { loom } \\
\text { weights of } \\
\text { total } \\
\end{array}$ \\
\hline Gaiano & MBA2-RBA1 & c. $1 \mathrm{ha}$ & 310 & 39 & 18 & 0 & 57 & 31.6 & 0.0 \\
\hline S. Pietro in Isola & MBA1-MBA2 & c. 1 ha & 180 & 48 & 28 & 1 & 77 & 36.4 & 1.3 \\
\hline $\begin{array}{l}\text { Castiglione di } \\
\text { Marano }\end{array}$ & MBA2-RBA1 & c. 0,8 ha & 158 & 82 & 19 & 0 & 101 & 18.8 & 0.0 \\
\hline Ca' de' Monesi & MBA2-RBA & c. $1 / 2$ ha & 156 & 30 & 15 & 0 & 45 & 33.3 & 0.0 \\
\hline Gorzano & MBA2-RBA2 & c. 0,8 ha & 155 & 680 & 443 & 73 & 1196 & 37.0 & 6.1 \\
\hline Castellarano & MBA2-MBA3 & unknown & 150 & 14 & 19 & 0 & 33 & 57.6 & 0.0 \\
\hline Pontenuovo & MBA2-RBA2 & c. 1 ha? & 140 & 104 & 7 & 0 & 111 & 6.3 & 0.0 \\
\hline S. Anastasio & MBA2-RBA1 & c. $1 \mathrm{ha}$ & 118 & 101 & 16 & 0 & 117 & 13.7 & 0.0 \\
\hline Bazzano & MBA2-early RBA1 & c. $1 \mathrm{ha}$ & 110 & 76 & 10 & 0 & 86 & 11.6 & 0.0 \\
\hline Monte Barello & MBA2-RBA1 & c. $1 / 2 \mathrm{ha}$ & 103 & 104 & 36 & 1 & 141 & 25.5 & 0.7 \\
\hline Trinità & MBA2-RBA1 & c. $1 \mathrm{ha}$ & 101 & 47 & 67 & 0 & 114 & 58.8 & 0.0 \\
\hline Pragatto & not available & not available & 70 & 162 & 43 & 3 & 208 & 20.7 & 1.4 \\
\hline Montale & MBA2-RBA2 & c. 1 ha & 65 & 1303 & 4454 & 127 & 5884 & 75.7 & 2.2 \\
\hline Casinalbo (abitato) & MBA2-RBA2 & c. 2 ha & 65 & 714 & 94 & 28 & 836 & 11.2 & 3.3 \\
\hline Gazzade & MBA1-RBA2 & c. 1 ha & 50 & 105 & 25 & 4 & 134 & 18.7 & 3.0 \\
\hline Savana di Cibeno & MBA2-RBA2 & c. $3 \mathrm{ha}$ & 29 & 25 & 4 & 0 & 29 & 13.8 & 0.0 \\
\hline Rastellino & MBA1-RBA1 & c. 3 ha & 27 & 89 & 40 & 0 & 129 & 31.0 & 0.0 \\
\hline Redù & MBA1-RBA2 & $\begin{array}{c}\text { c. } 2 \text { ha (MBA1-2), } \\
\text { c. } 12-14 \text { ha (MBA3- } \\
\text { RBA2) }\end{array}$ & 25 & 591 & 105 & 18 & 714 & 14.7 & 2.5 \\
\hline
\end{tabular}




\section{Plan of the site of Montale (Modena province), Italy}

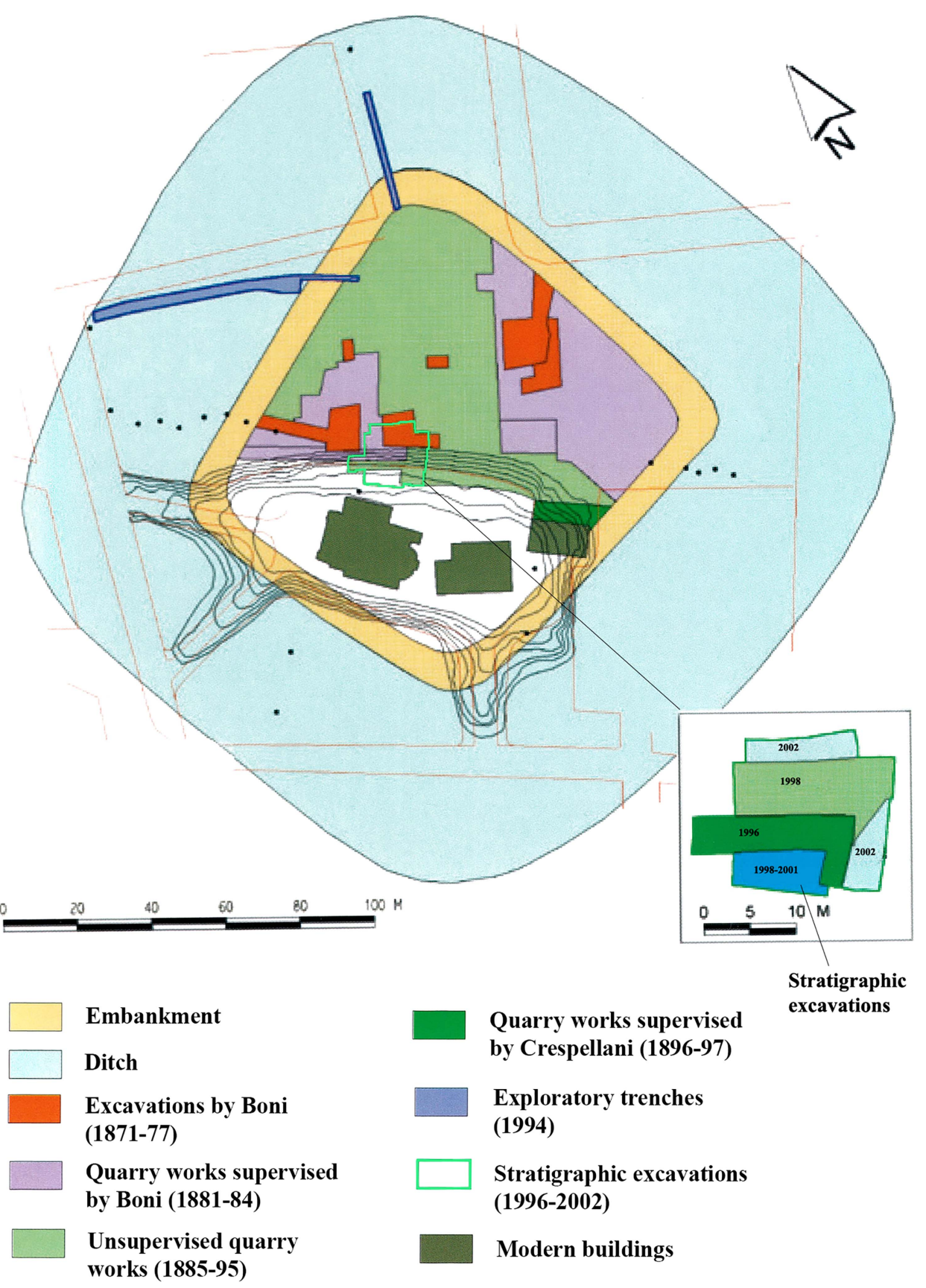

Fig. 3.

Plan of the site of Montale with excavation history (elaborated from Cardarelli 2009b, fig. 9)

that this phase was not only the best preserved, but also the one with larger portions of dwelling structures than other phases.
Here we present the analysis of the weight of the whorls, which seem to encompass a wide range of values (see below) with some chronological patterning. 
TABLE 3: WHOLE SPINDLE WHORLS FROM THE STRATIGRAPHIC EXCAVATIONS AT MONTALE PER PHASE (N=84)

\begin{tabular}{|c|c|c|c|c|c|c|c|c|c|}
\hline No & Year & Phase & Chronology & $\begin{array}{l}\text { Est. original } \\
\text { weight }(g)\end{array}$ & $\begin{array}{c}\text { Actual } \\
\text { weight }(g)\end{array}$ & $\begin{array}{l}\operatorname{Max.} \varnothing \\
(\mathrm{cm})\end{array}$ & $\begin{array}{l}\text { Height } \\
(\mathrm{cm})\end{array}$ & $\begin{array}{l}\varnothing \text { bole } \\
(\mathrm{cm})\end{array}$ & Type \\
\hline SWM1 & 2001 & $\mathrm{I} b$ & MBA2A & 15.2 & 15.2 & 3 & 1.9 & $0.5-0.6$ & 2a biconical asymmetric \\
\hline SWM2 & 2001 & $\mathrm{I} b$ & MBA2A & 10.9 & 10.9 & 3 & 1.3 & $0.5-0.5$ & 2a biconical asymmetric \\
\hline SWM3 & 2001 & $\mathrm{I} b$ & MBA2A & 17.4 & 17.4 & 3.1 & 1.9 & $0.5-0.6$ & 1a truncated conical \\
\hline SWM4 & 2001 & I b & MBA2A & 20.4 & 20.4 & 3.6 & 1.9 & $0.6-0.7$ & 1a truncated conical \\
\hline SWM5 & 2001 & I c & MBA2A & 12.9 & 12.9 & 3.4 & 1.4 & 0.6 & 2a biconical asymmetric \\
\hline SWM6 & 2001 & I c & MBA2A & 15.9 & 15.9 & 3.4 & 2.3 & 0.5 & $\begin{array}{l}3 \text { biconical asymmetric with plastic } \\
\text { protuberances }\end{array}$ \\
\hline SWM7 & 2001 & I c & MBA2A & 37 & 36.2 & 4.8 & 2.2 & 0.6 & 2a biconical asymmetric \\
\hline SWM8 & 2001 & I d & MBA2A & 15.2 & 15.2 & 3.3 & 1.5 & 0.8 & 2a biconical asymmetric \\
\hline SWM9 & 2001 & I d & MBA2A & 18.6 & 18.6 & 3.3 & 1.7 & $0.5-0.6$ & 2a biconical asymmetric \\
\hline SWM10 & 2001 & I d & MBA2A & 19.5 & 19.5 & 3.4 & 2.5 & 0.6 & 2a biconical asymmetric \\
\hline SWM11 & 2001 & I d & MBA2A & 17.1 & 17.1 & 3.3 & 2.7 & $0.5-0.6$ & 2a biconical asymmetric \\
\hline SWM12 & 2001 & I d & MBA2A & 10.1 & 10.1 & 2.9 & 1.8 & 0.6 & $\begin{array}{l}3 \text { biconical asymmetric with plastic } \\
\text { protuberances }\end{array}$ \\
\hline SWM13 & 2001 & II a & MBA2A & 15.4 & 15.4 & 3 & 1.7 & 0.6 & 2a biconical asymmetric (with decoration) \\
\hline SWM14 & 2001 & II a & MBA2A & 14.1 & 14.1 & 2.9 & 2.2 & 0.5 & 1a truncated conical \\
\hline SWM15 & 2001 & II a & MBA2A & 14.5 & 14.5 & 2.8 & 2 & $0.55-0.6$ & 2a biconical asymmetric \\
\hline SWM16 & 2001 & II a & MBA2A & 17.5 & 17.1 & 3.1 & 2 & 0.6 & $1 \mathrm{~b}$ truncated conical embossed profile \\
\hline SWM17 & 2001 & II a & MBA2A & 18.9 & 18.9 & 3.4 & 2.4 & 0.6 & $\begin{array}{l}3 \text { biconical asymmetric with plastic } \\
\text { protuberances }\end{array}$ \\
\hline SWM18 & 2001 & II $b$ & MBA2A & 12.8 & 12.8 & 3.2 & 2 & $0.5-0.6$ & $\begin{array}{l}3 \text { biconical asymmetric with plastic } \\
\text { protuberances }\end{array}$ \\
\hline SWM19 & 2001 & II $b$ & MBA2A & 20.4 & 20.4 & 4.1 & 1.3 & 0.4 & $2 \mathrm{~b}$ biconical asymmetric flattened \\
\hline SWM20 & 2001 & II b & MBA2A & 18 & 17.2 & 3.4 & 2.5 & $0.7-0.8$ & 1a truncated conical \\
\hline SWM21 & 2001 & II $b$ & MBA2A & 18.4 & 18.4 & 3.9 & 1.8 & 0.6 & $\begin{array}{l}\text { 1b truncated conical embossed profile (with } \\
\text { decoration) }\end{array}$ \\
\hline SWM22 & 2000 & II c & MBA2B & 24.1 & 24.1 & 3.9 & 2.2 & 0.6 & 2a biconical asymmetric \\
\hline SWM23 & 2001 & II c & MBA2B & 11.7 & 11.7 & 2.2 & 1.7 & 0.5 & 2a biconical asymmetric \\
\hline SWM24 & 2000 & II c & MBA2B & 13.5 & 13.5 & 3.2 & 1.5 & $0.5-0.6$ & 2a biconical asymmetric \\
\hline SWM25 & 2000 & II c & MBA2B & 14 & 14 & 2.5 & 2.2 & 0.5 & 4a biconical \\
\hline SWM26 & 2000 & II c & MBA2B & 14.4 & 14.4 & 2.8 & 2.5 & $0.6-0.7$ & $5 \mathrm{~b}$ biconical with concentric marks on 2 cones \\
\hline SWM27 & 2000 & II c & MBA2B & 8.9 & 8.9 & 2.3 & 2.2 & 0.5 & 5 a biconical with concentric marks on 1 cone \\
\hline SWM28 & 2002 & II c & MBA2B & 17 & 17 & 3.1 & 1.7 & 0.6 & 2a biconical asymmetric \\
\hline SWM29 & 2000 & II c & MBA2B & 17.2 & 17.2 & 3.2 & 1.7 & 0.5 & 2a biconical asymmetric \\
\hline SWM30 & 2000 & II c & MBA2B & 13.3 & 13.3 & 2.9 & 1.7 & $0.6-0.7$ & 2a biconical asymmetric \\
\hline SWM31 & 2000 & II c & MBA2B & 14 & 13.9 & 2.3 & 2.6 & $0.6-0.7$ & 5 a biconical with concentric marks on 1 cone \\
\hline SWM32 & 2000 & II c & MBA2B & 8.3 & 8.3 & 2.2 & 2.3 & $0.45-0.5$ & 5a biconical with concentric marks on 1 cone \\
\hline SWM33 & 2000 & II c & MBA2B & 11 & 9.9 & 2.6 & 1.9 & $0.5-0.6$ & 4a biconical \\
\hline SWM34 & 2001 & II c & MBA2B & 10.6 & 10.6 & 3.2 & 1.7 & 0.5 & $\begin{array}{l}1 \mathrm{~b} \text { truncated conical (with plastic decoration/ } \\
\text { protuberances) }\end{array}$ \\
\hline SWM35 & 2000 & II c & MBA2B & 11 & 11 & 2.5 & 1.9 & 0.55 & 2a biconical asymmetric \\
\hline SWM36 & 2000 & II c & MBA2B & 17.9 & 17.9 & 3.2 & 1.7 & 0.6 & 6 convex-cylindrical \\
\hline SWM37 & 2000 & III a & MBA3A & 11.3 & 11.3 & 2.8 & 1.8 & $0.4-0.6$ & $1 \mathrm{~b}$ truncated conical embossed profile \\
\hline
\end{tabular}


TABLE 3: (Continued)

\begin{tabular}{|c|c|c|c|c|c|c|c|c|c|}
\hline No & Year & Phase & Chronology & $\begin{array}{l}\text { Est. original } \\
\text { weight }(g)\end{array}$ & $\begin{array}{c}\text { Actual } \\
\text { weight (g) }\end{array}$ & $\begin{array}{c}\operatorname{Max} . \varnothing \\
(\mathrm{cm})\end{array}$ & $\begin{array}{l}\text { Height } \\
(\mathrm{cm})\end{array}$ & $\begin{array}{c}\varnothing \text { bole } \\
(\mathrm{cm})\end{array}$ & Type \\
\hline SWM38 & 2000 & III a & MBA3A & 19.2 & 19.2 & 3.1 & 2.1 & 0.6 & 4b biconical with embossed profile \\
\hline SWM39 & 2000 & III $b$ & MBA3A & 12.4 & 12.4 & 2.1 & 1.7 & 0.5 & 4b biconical with embossed profile \\
\hline SWM40 & 2000 & III $b$ & MBA3A & 15.6 & 13 & 2.6 & 2.2 & 0.5 & $5 \mathrm{~b}$ biconical with concentric marks on 2 cones \\
\hline SWM41 & 2000 & III $b$ & MBA3A & 14 & 14 & 2.7 & 2.9 & $0.5-0.6$ & 4a biconical \\
\hline SWM42 & 2000 & III $b$ & MBA3A & 14.1 & 14.1 & 3 & 2 & $0.5-0.6$ & 2a biconical asymmetric (with decoration) \\
\hline SWM43 & 1999 & III $b$ & MBA3A & 18 & 18 & 3.1 & 2.7 & 0.6 & 2a biconical asymmetric \\
\hline SWM44 & 2000 & III $b$ & MBA3A & 13.4 & 13.4 & 2.5 & 2.5 & 0.5 & 4a biconical \\
\hline SWM45 & 2000 & III c & MBA3A & 10 & 9.7 & 2.4 & 2.3 & 0.5 & $4 \mathrm{a}$ biconical \\
\hline SWM46 & 2000 & III c & MBA3A & 13.3 & 13.3 & 2.5 & 2.5 & $0.5-0.6$ & 4a biconical \\
\hline SWM47 & 2000 & III c & MBA3A & 17.4 & 17.4 & 3.1 & 1.9 & $0.5-0.6$ & 4a biconical \\
\hline SWM48 & 2000 & III $\mathrm{c}$ & MBA3A & 18.5 & 18.5 & 3.6 & 1.9 & $0.6-0.7$ & 2a biconical asymmetric \\
\hline SWM49 & 2000 & III c & MBA3A & 14.5 & 14.5 & 2.8 & 2.6 & 0.5 & 5 a biconical with concentric marks on 1 cone \\
\hline SWM50 & 2000 & III c & MBA3A & 17.4 & 17.4 & 3.1 & 1.9 & $0.5-0.6$ & 4a biconical \\
\hline SWM51 & 2000 & III c & MBA3A & 14.5 & 14.1 & 2.8 & 2.5 & 0.5 & 5a biconical with concentric marks on 1 cone \\
\hline SWM52 & 2000 & III c & MBA3A & 11.9 & 11.9 & 2.5 & 2.2 & 0.6 & 5 a biconical with concentric marks on 1 cone \\
\hline SWM53 & 1999 & IV a & MBA3A & 9 & 8.8 & 2.4 & 2.1 & $0.6-0.65$ & 5a biconical with concentric marks on 1 cone \\
\hline SWM54 & 1999 & IV a & MBA3A & 9.8 & 9.8 & 2.4 & 1.9 & $0.5-0.57$ & 5a biconical with concentric marks \\
\hline SWM55 & 1999 & IV a & MBA3A & 15.9 & 15.9 & 3 & 1.8 & $0.5-0.57$ & $4 \mathrm{~b}$ biconical with embossed profile \\
\hline SWM56 & 1999 & IV c & MBA3A & 19.3 & 19.3 & 2.9 & 2.2 & 0.5 & 8 globular (diagonal hole) \\
\hline SWM57 & 1999 & IV c & MBA3A & 12.7 & 12.7 & 3 & 1.4 & 0.6 & 6 convex-cylindrical \\
\hline SWM58 & 1999 & IV c & MBA3A & 13 & 13 & 2.5 & 2.3 & $0.5-0.6$ & 4a biconical \\
\hline SWM59 & 1999 & $\mathrm{VIb}$ & $\mathrm{MBA} 3 \mathrm{~A}+\mathrm{MBA3B}$ & 10 & 9.6 & 2.9 & 1.8 & 0.6 & 4 a biconical \\
\hline SWM60 & 1999 & VIb & $\mathrm{MBA} 3 \mathrm{~A}+\mathrm{MBA} 3 \mathrm{~B}$ & 10.7 & 10.7 & 2.4 & 2.3 & 0.6 & 4a biconical \\
\hline SWM61 & 1999 & VIb & MBA3A + MBA3B & 17 & 11.4 & 2.8 & 1.7 & $0.6-0.7$ & 5a biconical with concentric marks on 1 cone \\
\hline SWM62 & 1999 & VIb & $\mathrm{MBA} 3 \mathrm{~A}+\mathrm{MBA} 3 \mathrm{~B}$ & 11.9 & 11.9 & 2.4 & 2.2 & $0.5-0.6$ & 4a biconical \\
\hline SWM63 & 1999 & $\mathrm{VIb}$ & $\mathrm{MBA} 3 \mathrm{~A}+\mathrm{MBA} 3 \mathrm{~B}$ & 13 & 12.4 & 3 & 2 & 0.5 & 1a truncated conical \\
\hline SWM64 & 1999 & $\mathrm{VIb}$ & MBA3A + MBA3B & 13 & 12.4 & 2.7 & 2.3 & 0.5 & 5 a biconical with concentric marks on 1 cone \\
\hline SWM65 & 1999 & VIb & MBA3A + MBA3B & 12.5 & 12.5 & 2.5 & 2 & $0.55-0.6$ & 4a biconical \\
\hline SWM66 & 1999 & $\mathrm{VIb}$ & $\mathrm{MBA} 3 \mathrm{~A}+\mathrm{MBA} 3 \mathrm{~B}$ & 16.6 & 16.6 & 2.9 & 2.6 & 0.5 & 5a biconical with concentric marks on 1 cone \\
\hline SWM67 & 1999 & VIb & $\mathrm{MBA} 3 \mathrm{~A}+\mathrm{MBA3B}$ & 18.9 & 18.9 & 3.1 & 2 & 0.6 & 2a biconical asymmetric \\
\hline SWM68 & 1999 & VIb & MBA3A + MBA3B & 23.8 & 23.8 & 3.5 & 1.8 & 0.6 & 4b biconical with embossed profile \\
\hline SWM69 & 1999 & VIIb & MBA3B & 9.3 & 9.3 & 2.3 & 2.1 & 0.5 & 5 a biconical with concentric marks on 1 cone \\
\hline SWM70 & 1999 & VIIb & MBA3B & 10.5 & 10.5 & 2.7 & 1.5 & 0.5 & 2a biconical asymmetric \\
\hline SWM71 & 1999 & VIIb & MBA3B & 13.1 & 13.1 & 2.9 & 2.6 & 0.6 & 2a biconical asymmetric \\
\hline SWM72 & 1999 & VIIc & MBA3B & 13.1 & 13.1 & 2.7 & 2.3 & 0.5 & 2a biconical asymmetric \\
\hline SWM73 & 1999 & VIIc & MBA3B & 13.8 & 13.8 & 3.2 & 1.6 & $0.5-0.55$ & 2c biconical asymmetric with embossed profile \\
\hline SWM74 & 1999 & VIIc & MBA3B & 17.7 & 17.7 & 3.4 & 2 & 0.6 & 1a truncated conical \\
\hline SWM75 & 1999 & VIIIb & MBA3B & 10.7 & 10.7 & 2.8 & 2 & 0.5 & $2 \mathrm{a}$ biconical asymmetric \\
\hline SWM76 & 1999 & VIIIb & MBA3B & $15 ?$ & 14.3 & 3.8 & 2 & 0.7 & 9 pin-head shaped \\
\hline
\end{tabular}




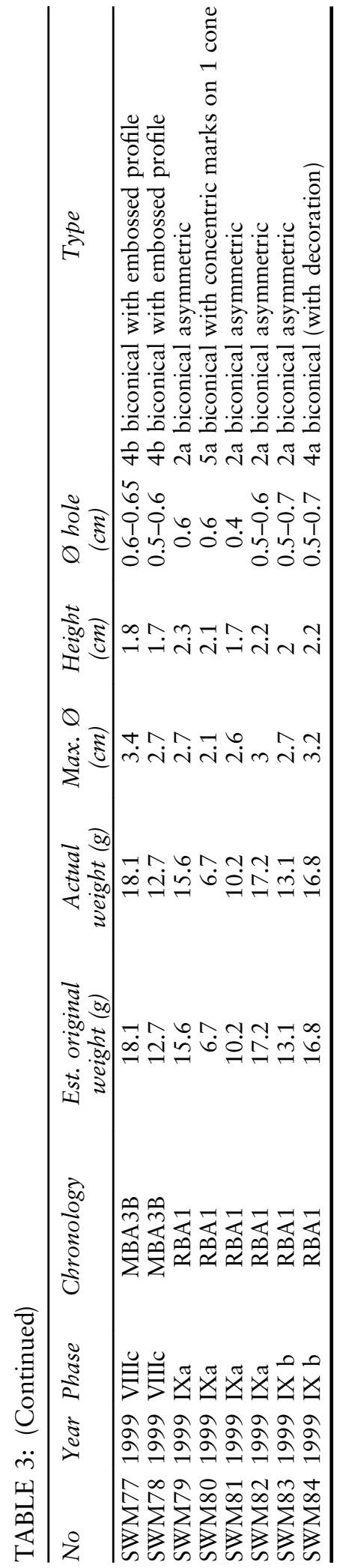

Ethnographical and experimental records suggest that weight and, to a certain extent, diameter, rather than shape, are functionally important for spinning. Bearing in mind that the chosen raw material might also influence both spinning techniques and spindle whorl sizes (eg, Barber 1991, 42-4; Siennika 2014, 165), light spindle whorls, under $10 \mathrm{~g}$, seem generally best to spin fine/light threads, whilst heavier whorls are more suited to thicker or coarser threads (Liu 1978; Barber 1991, 51-3; Olofsson et al. 2015) or for plying (Gleba 2008, 106). Although recent experiments have questioned these relationships, suggesting that the skills of spinners might be more important (Kania 2013), we believe that the analyses of weights can profitably initiate functional discussions. The material has been grouped at $10 \mathrm{~g}$ intervals, in accordance with recent experiments (Olofsson et al. 2015, 86-7), to provide a framework for further analyses. Among specimens collected during the 19th century, the lightest spindle whorls weight was as little as $1 \mathrm{~g}$, the heaviest, $85.5 \mathrm{~g}$; the majority of whorls (almost $70 \%$ of the total, 2848 pieces) weigh 10-20g (Fig. 5).

Of the well-dated 84 complete whorls recovered from modern excavations (Table 3), the lightest whorl weighs $6.7 \mathrm{~g}$ and the heaviest, $37 \mathrm{~g}$; as in the earlier collections, the majority of whorls were $10-20 \mathrm{~g}$ (Fig. 6). We have also attempted to correlate the weights with their diameters among the stratigraphically excavated whorls. The analyses of this sample suggests some diachronic differences, although counts are small. The scatter plot for diameter/weight of the material (Fig. 7) shows that a positive correlation apparently exists and that the larger and heavier whorls characterise the earliest period (Phase I), while (with the exception of Phases II and VI) any other period includes only items below $20 \mathrm{~g}$ and the largest number of whorls of $30 \mathrm{~mm}$ or less in diameter. Perhaps a craft/tradition prefering large whorls $(>30 \mathrm{~mm}$ in diameter) occurred mostly in Phases I and II. Finally, the greatest variety of spindle whorls, in terms of both weight and size, appears in Phase II, which might be a sign of more technological experimentation. In general, the great majority of the whorls fall between $c .10 \mathrm{~g}$ and $20 \mathrm{~g}$ (see also Figs 5-6) suggesting that a rather stable crafting tradition existed at Montale through time, although the frequency of spinning may have changed.

Diachronic analysis of spindle whorls from modern excavations, both whole (Table 3) and fragmentary (Table 4), shows that c. 50\% (52 whole and 41 
THE PREHISTORIC SOCIETY

TABLE 4: FRAGMENTARY SPINDLE WHORLS FROM THE STRATIGRAPHIC EXCAVATIONS AT MONTALE PER PHASE

\begin{tabular}{|c|c|c|c|c|c|c|c|c|}
\hline No. & Excavation data & Phase & Chronology & $\begin{array}{l}\text { Actual weight } \\
(g)\end{array}$ & $\begin{array}{l}\operatorname{Max} . \\
\varnothing(\mathrm{cm})\end{array}$ & Height $(\mathrm{cm})$ & $\begin{array}{l}\varnothing \text { bole } \\
(\mathrm{cm})\end{array}$ & Observations $=$ Type \\
\hline SWM85 & US3406 G12-13 13-07-01 & $\mathrm{Ib}$ & MBA2A & 7.5 & - & 2.1 & - & $\begin{array}{l}\text { c. } 1 / 2 \text { missing - 2a BIC. } \\
\text { ASYMM. }\end{array}$ \\
\hline SWM86 & US546 F11 05-07-99 & I c & MBA2A & 6 & 2.65 & - & - & $\begin{array}{l}\text { c. } 3 / 4 \text { missing }-1 \mathrm{c} \\
\text { TRUNC. BELL } \\
\text { SHAPED }\end{array}$ \\
\hline SWM87 & US3009 H12-13 22-06-01 & I c & MBA2A & 6.2 & 2.35 & 2.2 & 0.45 & $\begin{array}{l}\text { c. } 1 / 2 \text { missing - } 3 \text { BIC. } \\
\text { ASYMM. PROTUBER. }\end{array}$ \\
\hline SWM88 & US3270 G.H12 29-06-01 & I c & MBA2A & 6.4 & 3.3 & 1.95 & 0.5 & $\begin{array}{l}\text { c. } 2 / 3 \text { missing - } 2 \mathrm{a} \text { BIC. } \\
\text { ASYMM. }\end{array}$ \\
\hline SWM89 & US3295 E12 29-06-01 & I c & MBA2A & 3.4 & - & 1.6 & - & $\begin{array}{l}\text { fragmentary - 2c BIC. } \\
\text { ASYM. EMB. PROF. }\end{array}$ \\
\hline SWM90 & US3295 E 12 02-07-01 & I c & MBA2A & 9.5 & 3.2 & 1.8 & 0.55 & $\begin{array}{l}\text { c. } 1 / 2 \text { missing - 2c BIC. } \\
\text { ASYM. EMB. PROF. }\end{array}$ \\
\hline SWM91 & US 3008 G10-11 08-05-01 & I d & MBA2A & 5.2 & 3.2 & 1.8 & 0.5 & $\begin{array}{l}\text { fragmentary - 1a } \\
\text { TRUNC. CONICAL }\end{array}$ \\
\hline SWM92 & sotto us2022 E12-13 17-05-01 & $\mathrm{I}-\mathrm{II}$ a & MBA2A & 11.5 & 2.95 & 2.2 & 0.6 & $\begin{array}{l}\text { c. } 1 / 3 \text { missing }-2 \mathrm{a} \\
\text { BICONIC. ASYMM. }\end{array}$ \\
\hline SWM93 & US2652 F8 21-05-01 & II a & MBA2A & 3.2 & - & 1.6 & - & $\begin{array}{l}\text { fragmentary - 2c BIC. } \\
\text { ASYM. EMB. PROF. }\end{array}$ \\
\hline SWM94 & US2682 G7-8 24-05-01 & II a & MBA2A & 9.1 & 3.2 & 1.2 & 0.5 & $\begin{array}{l}\text { c. } 1 / 2 \text { missing - } 2 \mathrm{a} \text { BIC. } \\
\text { ASYMM. }\end{array}$ \\
\hline SWM95 & US2434 E6 23-04-01 & II b & MBA2A & 6.2 & 2.9 & 1.95 & 0.5 & $\begin{array}{l}\text { fragmentary - 2a BIC. } \\
\text { ASYM. }\end{array}$ \\
\hline SWM96 & US2314 F.G10 19-04-01 & II b & MBA2A & 6.3 & 2.8 & 2 & 0.4 & $\begin{array}{l}\text { c. } 1 / 2 \text { missing }-8 \\
\text { GLOBULAR }\end{array}$ \\
\hline SWM97 & US2209 E5 -2001 & II $\mathrm{c}$ & MBA2B & 3 & - & - & - & fragmentary - TYPE? \\
\hline SWM98 & US3385 G8 09-07-01 & II $\mathrm{c}$ & MBA2B & 33.2 & 5.7 & 2.7 & 1 & $\begin{array}{l}\text { fragmentary - 2c BIC. } \\
\text { ASYM. EMBOS. } \\
\text { PROF. }\end{array}$ \\
\hline SWM99 & US2203 F7 24-10-00 & II c & MBA2B & 4.5 & 3 & 2 & 0.4 & $\begin{array}{l}\text { fragmentary - 4b BIC. } \\
\text { EMBOSS. PROF.? }\end{array}$ \\
\hline SWM100 & US2176 E7 18-10-00 & II $\mathrm{c}$ & MBA2B & 27.4 & 4.65 & 3.2 & 0.8 & $\begin{array}{l}\text { c. } 1 / 2 \text { missing - 2c BIC. } \\
\text { ASYM. EMB. PROF. }\end{array}$ \\
\hline SWM101 & US2014 H9 15-09-00 & II c & MBA2B & 1.7 & - & - & - & $\begin{array}{l}\text { fragmentary - 5b BIC. } \\
\text { CONCENTRIC } \\
\text { MARKS } 2 \text { CONES }\end{array}$ \\
\hline SWM102 & US2148 F13 20-09-00 & II c & MBA2B & 6.3 & 2.9 & - & 0.5 & $\begin{array}{l}\text { fragmentary - 1c BELL } \\
\text { SHAPED (+ } \\
\text { decoration) }\end{array}$ \\
\hline SWM103 & US2085 H.G12 21-09-? & II c & MBA2B & 5.7 & 3.1 & - & 0.4 & $\begin{array}{l}\text { c. } 1 / 2 \text { missing - } 3 \text { BIC. } \\
\text { ASYM. PROTUBER. }\end{array}$ \\
\hline SWM104 & US2277 G13-14 31-10-00 & II $\mathrm{c}$ & MBA2B & 7.8 & 3.1 & 1.9 & 0.4 & $\begin{array}{l}\text { c. } 1 / 2 \text { missing - } 2 \mathrm{~b} \text { BIC. } \\
\text { ASYM. FLATTENED }\end{array}$ \\
\hline SWM105 & US2014 G13 14-09-00 & II c & MBA2B & 6.1 & 3 & 2.45 & 0.5 & $\begin{array}{l}\text { c. } 2 / 3 \text { missing - 2c BIC. } \\
\text { ASYM. EMB. PROF. }\end{array}$ \\
\hline SWM106 & US2014 F11 14-09-00 & II c & MBA2B & 3.5 & 2.35 & - & 0.5 & $\begin{array}{l}\text { fragmentary - 5a BIC. } \\
\text { CONCENTRIC } \\
\text { MARKS } 1 \text { CONE }\end{array}$ \\
\hline SWM107 & US2014 H9 18-09-00 & II c & MBA2B & 2.9 & - & - & - & $\begin{array}{c}\text { fragmentary - 5a BIC. } \\
\text { CONCENTRIC } \\
\text { MARKS } 1 \text { CONE }\end{array}$ \\
\hline SWM108 & US2130 F.G11 -2000 & II $\mathrm{c}$ & MBA2B & 6.1 & 2.7 & 1.55 & 0.6 & $\begin{array}{l}\text { c. } 1 / 2 \text { missing - } 6 \mathrm{CO} \\
\text { NVEX- } \\
\text { CYLINDRICAL? }\end{array}$ \\
\hline SWM109 & US2302 G9 13-04-01 & II c & MBA2B & 10.7 & 4.2 & 1.8 & $0.9 *$ & $\begin{array}{l}\text { "1 frag. another too small } \\
\text { to measure } c .1 / 2 \\
\text { missing } \\
-6 \text { CONVEX- } \\
\text { CYLINDRICAL? }\end{array}$ \\
\hline SWM110 & US2336 G8 17-04-01 & II $\mathrm{c}$ & & 9.6 & - & - & - & fragmentary - TYPE? \\
\hline & & & & & 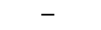 & - & - & fragmentary - TYPE? \\
\hline $\begin{array}{l}\text { SWM112 } \\
\text { SWM113 }\end{array}$ & US788 \& 963 E7-8 29-09-00 & II-III & MBA2 + MBA3A & 2.1 & - & - & - & fragmentary - TYPE? \\
\hline SWM113 & US2002 G10 12-09-00 & III a & MBA3A & 3 & 2.2 & - & 0.45 & $\begin{array}{l}\text { fragmentary - 5b BIC. } \\
\text { CONCENTRIC } \\
\text { MARKS } 2 \text { CONES }\end{array}$ \\
\hline SWM114 & US761 I9 27-06-00 & III $b$ & MBA3A & 3.3 & 2.7 & 1.5 & 0.5 & $\begin{array}{l}\text { fragmentray - 2b BIC. } \\
\text { ASYMM. } \\
\text { FLATTENED }\end{array}$ \\
\hline SWM115 & US761 G5 28-06-00 & III b & MBA3A & 4.8 & 2.5 & 1.7 & 0.45 & \\
\hline
\end{tabular}


TABLE 4: (Continued)

\begin{tabular}{|c|c|c|c|c|c|c|c|c|}
\hline No. & Excavation data & Phase & Chronology & $\begin{array}{c}\text { Actual weight } \\
\text { (g) }\end{array}$ & $\begin{array}{l}\operatorname{Max} . \\
\varnothing(\mathrm{cm})\end{array}$ & Height $(\mathrm{cm})$ & $\begin{array}{c}\varnothing \text { bole } \\
(\mathrm{cm})\end{array}$ & Observations $=$ Type \\
\hline SWM116 & US761 H9 27-06-00 & III $b$ & MBA3A & 4.4 & 2.95 & - & 0.5 & $\begin{array}{l}\text { c. } 1 / 2 \text { missing - } \\
\text { IRREGULAR } \\
\text { fragmentray - } 1 \mathrm{c} \text { TRUNC } \\
\text { BELL SHAPED? }\end{array}$ \\
\hline SWM117 & US761 H9 27-06-00 & III $b$ & MBA3A & 2.9 & 2.2 & 1.65 & 0.4 & $\begin{array}{l}\text { c. } 2 / 3 \text { missing - 2c BIC. } \\
\text { ASYM. EMBOS. } \\
\text { PROF. }\end{array}$ \\
\hline SWM118 & US658 F14 20-07-00 & III $b$ & MBA3A & 4.5 & 2.5 & 2.6 & 0.5 & $\begin{array}{l}\text { c. } 2 / 3 \text { missing }-4 \mathrm{a} \\
\text { BICONICAL }\end{array}$ \\
\hline SWM119 & US906 H11 25-07-00 & III $b$ & MBA3A & 8.6 & 2.85 & 2.2 & 0.4 & $\begin{array}{l}\text { c. } 1 / 2 \text { missing - } 4 \mathrm{~b} \text { BIC. } \\
\text { EMBOS. PROF. }\end{array}$ \\
\hline SWM120 & US957 G10 03-08-2000 & III $b$ & MBA3A & 5.9 & 2.9 & 1.95 & 0.5 & $\begin{array}{l}\text { c. } 1 / 2 \text { missing }-3 \text { BIC. } \\
\text { ASYM. PROTUBER. }\end{array}$ \\
\hline SWM121 & US913 H14 31-07-00 & III $b$ & MBA3A & 3.1 & - & - & - & $\begin{array}{l}\text { fragmentray, irregular - } \\
\text { TYPE? }\end{array}$ \\
\hline SWM122 & US874 F13 25-07-00 & III b & MBA3A & 13.2 & 2.95 & 2.9 & 0.5 & $\begin{array}{l}\text { fragmentray }-4 a \\
\text { BICONICAL }\end{array}$ \\
\hline SWM123 & US906 E13-14 25-07-00 & III $b$ & MBA3A & 4.9 & 2.3 & - & 0.5 & $\begin{array}{l}\text { c. } 1 / 2 \text { missing - 5a BIC. } \\
\text { CONCENTRIC } \\
\text { MARKS } 1 \text { CONE }\end{array}$ \\
\hline SWM124 & US656 E11 10-07-00 & III $c$ & MBA3A & 7.6 & 2.6 & 2.8 & 0.45 & $\begin{array}{l}\text { c. } 1 / 2 \text { missing }-4 a \\
\text { BICONICAL }\end{array}$ \\
\hline SWM125 & US706 G9 29-06-00 & III $\mathrm{c}$ & MBA3A & 5.6 & 3 & 2.5 & 0.5 & $\begin{array}{l}\text { fragmentray - 5a BIC. } \\
\text { CONCENTRIC } \\
\text { MARKS } 1 \text { CONE }\end{array}$ \\
\hline SWM126 & US656 25-06-00 & III c & MBA3A & 9.9 & 2.95 & 2.4 & 0.6 & $\begin{array}{l}\text { c. } 1 / 2 \text { missing - } 4 \mathrm{a} \text { BIC. } \\
\text { (EMBOSS. PROF.?) }\end{array}$ \\
\hline SWM127 & US687 H11-12 1999 & IV a & MBA3A & 5.8 & 3 & - & 0.6 & $\begin{array}{l}\text { fragmentary - 2a BYC. } \\
\text { ASYMM. }\end{array}$ \\
\hline SWM128 & US697 F9 21-06-00 & IV a & MBA3A & 16.2 & 3.4 & 2.6 & 0.55 & $\begin{array}{l}\text { failed during manufacture } \\
\quad-4 a \text { BICONICAL }\end{array}$ \\
\hline SWM129 & US755 F5 21-06-00 & IV a & MBA3A & 7.7 & 3.1 & 2.2 & 0.55 & $\begin{array}{l}\text { c. } 1 / 2 \text { missing - 1c } \\
\text { TRUNC. BELL } \\
\text { SHAPED? }\end{array}$ \\
\hline SWM130 & US710 H.G9 22-06-00 & IV a & MBA3A & 5.6 & 2.9 & - & 0.5 & c. $2 / 3$ missing $-4 a$ \\
\hline SWM131 & 23-06-2000 & IV a & MBA3A & 6.5 & - & - & - & $\begin{array}{l}\text { very fragmentary - } \\
\text { TYPE? }\end{array}$ \\
\hline SWM132 & US741 F8 19-06-00 & IV c & MBA3A & 1.7 & - & - & - & $\begin{array}{l}\text { very fragmentary - } 4 a \\
\text { BICONICAL? }\end{array}$ \\
\hline SWM133 & US704 E6 20-06-00 & IV c & MBA3A & 4.2 & 2.4 & 2.35 & 0.4 & $\begin{array}{l}\text { c. } 2 / 3 \text { missing }-4 \mathrm{a} \\
\text { BICONICAL }\end{array}$ \\
\hline SWM134 & US641 G11 11-10-99 & IV c & MBA3A & 3.4 & - & - & - & $\begin{array}{l}\text { fragmentary - 4b BIC. } \\
\text { EMB. PROF.? }\end{array}$ \\
\hline SWM135 & US632 H5 15-10-99 & IV c & MBA3A & 4.9 & 2.8 & 1.9 & 0.5 & $\begin{array}{l}\text { c. } 2 / 3 \text { missing }-1 \mathrm{~b} \\
\text { TRUNC. CONICAL } \\
\text { EMB. PROF. }\end{array}$ \\
\hline SWM136 & US641 E10 12-10-99 & IV c & MBA3A & 4.3 & 2.7 & - & 0.5 & $\begin{array}{l}\text { c. } 2 / 3 \text { missing }-1 \mathrm{~b} \\
\text { TRUNC. CONICAL } \\
\text { EMB. PROF.? }\end{array}$ \\
\hline SWM137 & US641 06-10-99 & IV c & MBA3A & 5.3 & - & 2.7 & & $\begin{array}{l}\text { c. } 60 \% \text { missing }-4 \mathrm{a} \\
\text { BICONICAL }\end{array}$ \\
\hline SWM138 & US677 E.F6 21-10-99 & $\mathrm{Vb}$ & MBA3A & 4 & 2.4 & 2.4 & 0.4 & $\begin{array}{l}\text { c. } 2 / 3 \text { missing - } 4 \mathrm{a} \\
\text { BICONICAL }\end{array}$ \\
\hline SWM139 & US636 G6-7 16-09-99 & VI b & $\mathrm{MBA} 3 \mathrm{~A}+\mathrm{MBA} 3 \mathrm{~B}$ & 2.4 & - & - & - & fragmentary - TYPE? \\
\hline SWM140 & US 636 G6-7 16-09-99 & $\mathrm{VI} \mathrm{b}$ & $\mathrm{MBA} 3 \mathrm{~A}+\mathrm{MBA} 3 \mathrm{~B}$ & 7.4 & 2.8 & 2.6 & 0.6 & $\begin{array}{l}\text { c. } 1 / 2 \text { missing }(2 \mathrm{fr} .)-4 a \\
\text { BICONICAL }\end{array}$ \\
\hline SWM141 & US674 E7-8 12-10-99 & VI b & MBA3A + MBA3B & 3.8 & - & - & 0.5 & $\begin{array}{l}\text { fragmentary }-1 \mathrm{a} \\
\text { TRUNCATED } \\
\text { CONICAL }\end{array}$ \\
\hline SWM142 & US674 E8 12-10-99 & VI b & MBA3A + MBA3B & 3.5 & 2.4 & 2 & 0.4 & $\begin{array}{l}\text { c. } 3 / 4 \text { missing }-4 \mathrm{a} \\
\text { BICONICAL }\end{array}$ \\
\hline SWM143 & US674 E8 12-10-99 & VI b & MBA3A + MBA3B & 3.5 & - & - & 0.6 & fragmentary - TYPE? \\
\hline SWM144 & US593 F8 22-09-99 & VI b & $\mathrm{MBA} 3 \mathrm{~A}+\mathrm{MBA} 3 \mathrm{~B}$ & 7.8 & 2.6 & 1.95 & 0.5 & $\begin{array}{l}\text { c. } 1 / 2 \text { missing }-2 \mathrm{a} \\
\text { BICONIC. ASYM }\end{array}$ \\
\hline SWM145 & US593 F8 22-09-99 & VI b & $\mathrm{MBA} 3 \mathrm{~A}+\mathrm{MBA} 3 \mathrm{~B}$ & 6 & 2.45 & 2.3 & 0.45 & $\begin{array}{l}\text { c. } 1 / 2 \text { missing }-4 \mathrm{a} \\
\text { BICONICAL }\end{array}$ \\
\hline SWM146 & US593 F8 22-09-99 & VI b & MBA3A + MBA3B & 8.2 & 3 & - & 0.55 & $\begin{array}{l}\text { c. } 2 / 3 \text { \& bases missing - } \\
\text { 4a BICONICAL }\end{array}$ \\
\hline
\end{tabular}


TABLE 4: (Continued)

\begin{tabular}{|c|c|c|c|c|c|c|c|c|}
\hline No. & Excavation data & Phase & Chronology & $\begin{array}{c}\text { Actual weight } \\
(g)\end{array}$ & $\begin{array}{l}\text { Max. } \\
\varnothing(\mathrm{cm})\end{array}$ & Height $(\mathrm{cm})$ & $\begin{array}{l}\varnothing \text { bole } \\
(\mathrm{cm})\end{array}$ & Observations $=$ Type \\
\hline SWM147 & US607 H.i13 09-09-99 & VI b & MBA3A + MBA3B & 4.5 & 2.5 & 1.8 & 0.45 & $\begin{array}{l}\text { c. } 1 / 2 \text { missing }-5 \mathrm{a} \text { BIC. } \\
\text { CONCENTRIC } \\
\text { MARKS } 1 \text { CONE }\end{array}$ \\
\hline SWM148 & US 635 E.F 9-10 23-09-99 & VI b & $\mathrm{MBA} 3 \mathrm{~A}+\mathrm{MBA3B}$ & 4.7 & - & 2.8 & - & $\begin{array}{l}\text { c. } 2 / 3 \text { missing }-4 \mathrm{a} \\
\text { BICONICAL }\end{array}$ \\
\hline SWM149 & US593 F6 22-09-99 & VI b & $\mathrm{MBA} 3 \mathrm{~A}+\mathrm{MBA3B}$ & 7.1 & 2.8 & 2.15 & 0.5 & $\begin{array}{l}\text { c. } 1 / 2 \text { missing - } 4 \mathrm{~b} \text { BIC. } \\
\text { EMB. PROF. }\end{array}$ \\
\hline SWM150 & US593 H9 23-09-99 & VI b & $\mathrm{MBA} 3 \mathrm{~A}+\mathrm{MBA} 3 \mathrm{~B}$ & 4.5 & - & - & - & $\begin{array}{l}\text { fragmentary - 4a } \\
\text { BICONICAL }\end{array}$ \\
\hline SWM151 & US593 H9 23-09-99 & VI b & MBA3A + MBA3B & 2.9 & - & - & - & $\begin{array}{l}\text { fragmentary - 1a } \\
\text { TRUNCATED } \\
\text { CONICAL }\end{array}$ \\
\hline SWM152 & US593 F7 22-09-99 & VI b & MBA3A + MBA3B & 7.5 & 2.95 & 2.1 & 0.55 & $\begin{array}{l}\text { c. } 1 / 2 \text { missing ( } 2 \text { fr. })-4 b \\
\text { BICONIC. EMB. } \\
\text { PROF. }\end{array}$ \\
\hline SWM153 & US593 F7 22-09-99 & VI b & $\mathrm{MBA} 3 \mathrm{~A}+\mathrm{MBA} 3 \mathrm{~B}$ & 2.4 & - & 2 & - & $\begin{array}{l}\text { fragmentary }-4 a \\
\text { BICONICAL }\end{array}$ \\
\hline SWM154 & US583 F8 17-09-99 & VII a & MBA3B & 2.4 & - & - & - & $\begin{array}{l}\text { fragmentary - 2a } \\
\text { BICONIC. ASYM. }\end{array}$ \\
\hline SWM155 & US583 E8 24-09-99 & VII a & MBA3B & 3.2 & - & - & - & fragmentary - TYPE? \\
\hline SWM156 & UIS601 E6 07-09-99 & VII b & MBA3B & 1.9 & - & - & - & fragmentary - TYPE? \\
\hline SWM157 & US590 H14 22-07-99 & VII b & MBA3B & 8.1 & 2.85 & 2 & 0.55 & $\begin{array}{l}\text { c. } 1 / 2 \text { missing - } 2 \mathrm{a} \text { BIC. } \\
\text { ASYMM. }\end{array}$ \\
\hline SWM158 & US578 E.F7 20-07-99 & VII c & MBA3B & 7.9 & 2.95 & 2.2 & 0.5 & $\begin{array}{l}\text { c. } 1 / 2 \text { missing }-5 \text { a BIC. } \\
\text { CONCENTRIC } \\
\text { MARKS } 1 \text { CONE }\end{array}$ \\
\hline SWM159 & US582 G6 23-07-99 & VII c & MBA3B & 7 & 2.8 & 2.1 & 0.6 & $\begin{array}{l}\text { c. } 60 \% \text { missing - 2a BIC. } \\
\text { ASYMM. }\end{array}$ \\
\hline SWM160 & US582 F8 23-07-99 & VII c & MBA3B & 3 & - & - & - & fragmentary - TYPE? \\
\hline SWM161 & US621 F5 13-09-99 & VII c & MBA3B & 8.3 & 2.8 & 2.4 & 0.55 & $\begin{array}{l}\text { c. } 1 / 2 \text { missing - 4b } \\
\text { BICONIC. EMB. } \\
\text { PROF. }\end{array}$ \\
\hline SWM162 & US582 H9 07-09-99 & VII c & МВA3B & 10.6 & 3.55 & 2.85 & 0.5 & $\begin{array}{l}\text { c. } 55 \% \text { missing }-1 \mathrm{~b} \\
\text { TRUNC. CON. EMB. } \\
\text { PROF. }\end{array}$ \\
\hline SWM163 & US629 H6 09-09-99 & VII c & MBA3B & 7.5 & 2.95 & 1.85 & 0.5 & $\begin{array}{l}\text { c. } 1 / 2 \text { missing - 2c BIC. } \\
\text { ASYMM. EMB. PROF. }\end{array}$ \\
\hline SWM164 & US555 E6 29-06-99 & VIII a & MBA3B & 11.5 & 3.25 & 2.6 & 0.5 & $\begin{array}{l}\text { c. } 1 / 2 \text { missing - 4b BIC. } \\
\text { EMBOSS. PROF. }\end{array}$ \\
\hline SWM165 & US561 F7 06-09-99 & VIII a & MBA3B & 3.4 & - & 2 & - & $\begin{array}{l}\text { fragmentary - } 4 \mathrm{~b} \\
\text { BICONIC. EMB. } \\
\text { PROF. }\end{array}$ \\
\hline SWM166 & US548 E13 13-07-99 & VIII b & MBA3B & 14.3 & 3.95 & - & 0.7 & $\begin{array}{l}\text { fragmentary - } 9 \text { PIN- } \\
\text { HEAD }\end{array}$ \\
\hline SWM167 & US548 G14 12-07-99 & VIII b & MBA3B & 6 & 3.3 & - & 0.5 & $\begin{array}{l}\text { c. } 1 / 2 \text { missing }-1 \mathrm{c} \\
\text { TRUNC. BELL } \\
\text { SHAPED? }\end{array}$ \\
\hline SWM168 & US 548-558 G11 06-07-99 & VIII b & MBA3B & 9.1 & 2.8 & 2.5 & 0.6 & $\begin{array}{l}\text { c. } 1 / 2 \text { missing - 4b BIC. } \\
\text { EMBOSS. PROF. }\end{array}$ \\
\hline SWM169 & US548-558 E10 06-07-99 & VIII b & MBA3B & 8.9 & 3.2 & 1.9 & 0.45 & $\begin{array}{l}\text { c. } 1 / 2 \text { missing }-4 \mathrm{~b} \text { BIC. } \\
\text { EMBOSS. PROF.? }\end{array}$ \\
\hline SWM170 & US548-558 E10 06-07-99 & VIII b & MBA3B & 4.5 & - & - & - & fragmentary - TYPE? \\
\hline SWM171 & US546 G13-14 02-07-99 & VIII c & MBA3В & 8.4 & 2.9 & 2 & 0.4 & $\begin{array}{l}\text { c. } 1 / 2 \text { missing - } 4 \mathrm{~b} \text { BIC. } \\
\text { EMBOSS. PROF. }\end{array}$ \\
\hline SWM172 & US549 H8 IItagl. 28-06-99 & IX a & RBA1 & 3 & 3.1 & - & - & $\begin{array}{l}\text { Very fragmentary - } \\
\text { TYPE? }\end{array}$ \\
\hline SWM173 & US547 E8 IItagl. 01-07-99 & IX a & RBA1 & 4.1 & 2.4 & 1.7 & 0.45 & $\begin{array}{l}\text { c. } 1 / 2 \text { missing }-8 \\
\text { GLOBULAR }\end{array}$ \\
\hline SWM174 & US549 E6 28-06-99 & IX a & RBA1 & 7.7 & 2.4 & 2.7 & - & $\begin{array}{l}\text { c. } 1 / 2 \text { missing- WHORL? } \\
\text { TYPE? }\end{array}$ \\
\hline SWM175 & US550 H5-6 26-06-99 & IX a & RBA1 & 3.4 & 2.1 & - & - & $\begin{array}{l}\text { c. } 3 / 4 \text { missing - } 4 \mathrm{~b} \text { BIC. } \\
\text { EMB. PROF.? }\end{array}$ \\
\hline SWM176 & US550 H5 28-06-99 & IX a & RBA1 & 6.5 & 3 & 2.05 & 0.55 & $\begin{array}{l}\text { c. } 2 / 3 \text { missing - 2c BIC. } \\
\text { ASYM. EMBOSS. } \\
\text { PROF. }\end{array}$ \\
\hline SWM177 & US549 E.F5 28-06-99 & IX a & RBA1 & 5.8 & 3.15 & 1.8 & 0.6 & $\begin{array}{l}\text { c. } 1 / 2 \text { missing - 4b BIC. } \\
\text { EMBOSS. PROF.? }\end{array}$ \\
\hline SWM178 & US550 G6 28-06-99 & IX a & RBA1 & 8.4 & 2.95 & 2.4 & 0.6 & $\begin{array}{l}\text { c. } 1 / 2 \text { missing - 4b BIC. } \\
\text { EMBOSS. PROF. }\end{array}$ \\
\hline
\end{tabular}


TABLE 4: (Continued)

\begin{tabular}{|c|c|c|c|c|c|c|c|c|}
\hline No. & Excavation data & Phase & Chronology & $\begin{array}{c}\text { Actual weight } \\
\text { (g) }\end{array}$ & $\begin{array}{l}\operatorname{Max} . \\
\varnothing(\mathrm{cm})\end{array}$ & Height $(\mathrm{cm})$ & $\begin{array}{l}\varnothing \text { bole } \\
(\mathrm{cm})\end{array}$ & Observations $=$ Type \\
\hline SWM179 & US544 H4-5 23-06-99 & IX b & RBA1 & 5 & - & - & - & $\begin{array}{l}\text { c. } 3 / 4 \text { missing }-2 \mathrm{a} \\
\text { BICONIC. ASYMM. }\end{array}$ \\
\hline SWM180 & US542 F9-10 24-06-99 & IX b & RBA1 & 5.6 & 2.7 & 1.6 & 0.5 & $\begin{array}{l}\text { c. } 1 / 2 \text { missing }-1 \mathrm{c} \\
\text { TRUNC. BELL } \\
\text { SHAPED }\end{array}$ \\
\hline SWM181 & US542 H13 22-06-99 & IX b & RBA1 & 3.3 & 3.1 & - & 0.4 & $\begin{array}{l}\text { c. } 2 / 3 \text { missing }-1 \mathrm{c} \\
\text { TRUNC. BELL } \\
\text { SHAPED? }\end{array}$ \\
\hline SWM182 & US521 IIItagl. G.F 12 16-06-99 & $\mathrm{X}$ a & RBA1 & 10.3 & 3.5 & 2.85 & 0.5 & $\begin{array}{l}\text { c. } 3 / 4 \text { missing }-4 \mathrm{~b} \text { BIC. } \\
\text { EMB. PROF.? }\end{array}$ \\
\hline
\end{tabular}

fragmentary) belong to Phase I-III and that, with the exception of two fragmentary items, one from Phase $\mathrm{V}$ and one from Phase X, practically no whorls exist from Phase V, X, and XI. Small sample sizes, however, make trends unreliable. The lack of spindle whorls from Phase V, for example, clearly reflects that the small area excavated ceased to be a dwelling space during that phase as it was involved primarily in metallurgy. The lack of whorls in Phase X-XI might reflect poor preservation (Cardarelli 2009b, 45, 50-1, 63). Although detailed regional studies are needed, which could add important new information, the decrease of clay spindle whorls during the Recent Bronze Age may reflect a transformation in textile production modes or outcomes across the whole Terramare area. ${ }^{8}$

\section{DISCUSSION}

On the base of the available data (cf. Bernabò Brea et al. 2003; Bianchi 2004; Lincetto 2006, see also Table 2), the counts of clay spindle whorls from Montale seem to have no equal among Terramare settlements, nor in fact in other Bronze Age European settlements (cf. Gleba \& Mannering 2012; Grömer 2013; 2016; Kneisel \& Schaefer in press). For the Mediterranean, where written sources speak of intense production, the impressive database created by the Centre for Textile Research in Copenhagen, although far from being exhaustive, counts only a total of 3994 entries (Andersson Strand \& Nosch 2015b, 149), including known evidence from major Bronze Age centres around the Eastern Mediterranean coast. With the exception of Troy (Guzowska et al. 2015), spindle whorl counts from all the investigated sites do not exceed a few hundred (Andersson \& Nosch 2015). Therefore, the assemblage of thousands of spindle whorls from Montale strongly suggests that the settlement was unusual, specialising in intense yarn production. Although weight distributions (Figs $5 \& 6$ ) show wide variation, a significant presence of light whorls $(\leq 10 \mathrm{~g})$ and a clear dominance of medium-light spindle whorls $(10-20 \mathrm{~g})$ suggests the production of a variety of threads including thin or fine yarn. That thin yarn was manufactured or used on site has been suggested on the basis of the numerous needles from Montale with small eyes appropriate for thin threads (Pulini \& Righi 2009, 100).

Evidence from Montale suggests that specialised workers were probably active at the site and that production was on a large scale. As discussed earlier, spinning is a sizable task in textile production and so the high quantity of spindle whorls suggests their importance in the settlement's everyday activities. In this respect, the rather abrupt disappearence of whorls in Phases X-XI (Fig. 1) is difficult to explain, in particular when considering that, during these phases, the number of sheep/goats in the bone assemblage increased (Table 1). Although the representativeness of the excavated collection for the whole site is unknown, one can propose that more intense yarn production occurred during the Middle Bronze Age followed by increasing diversification of productive activities in later phases. One reasonable suggestion is that, at the onset of Montale Phase X, emphasis on raw wool as an export became more profitable than yarn production, perhaps indicating some transformations of regional management practices or an emerging trade in other products. Archaeobotanical samples from modern excavations suggest that, at Montale, another economic transformation occurred at the end of Phase VIII and more evidently in Phase IX. With the introduction of grape there (Accorsi et al. 2009, 67; Cardarelli et al. forthcoming), perhaps new products, such as wine, might have replaced spinning as Montale's 

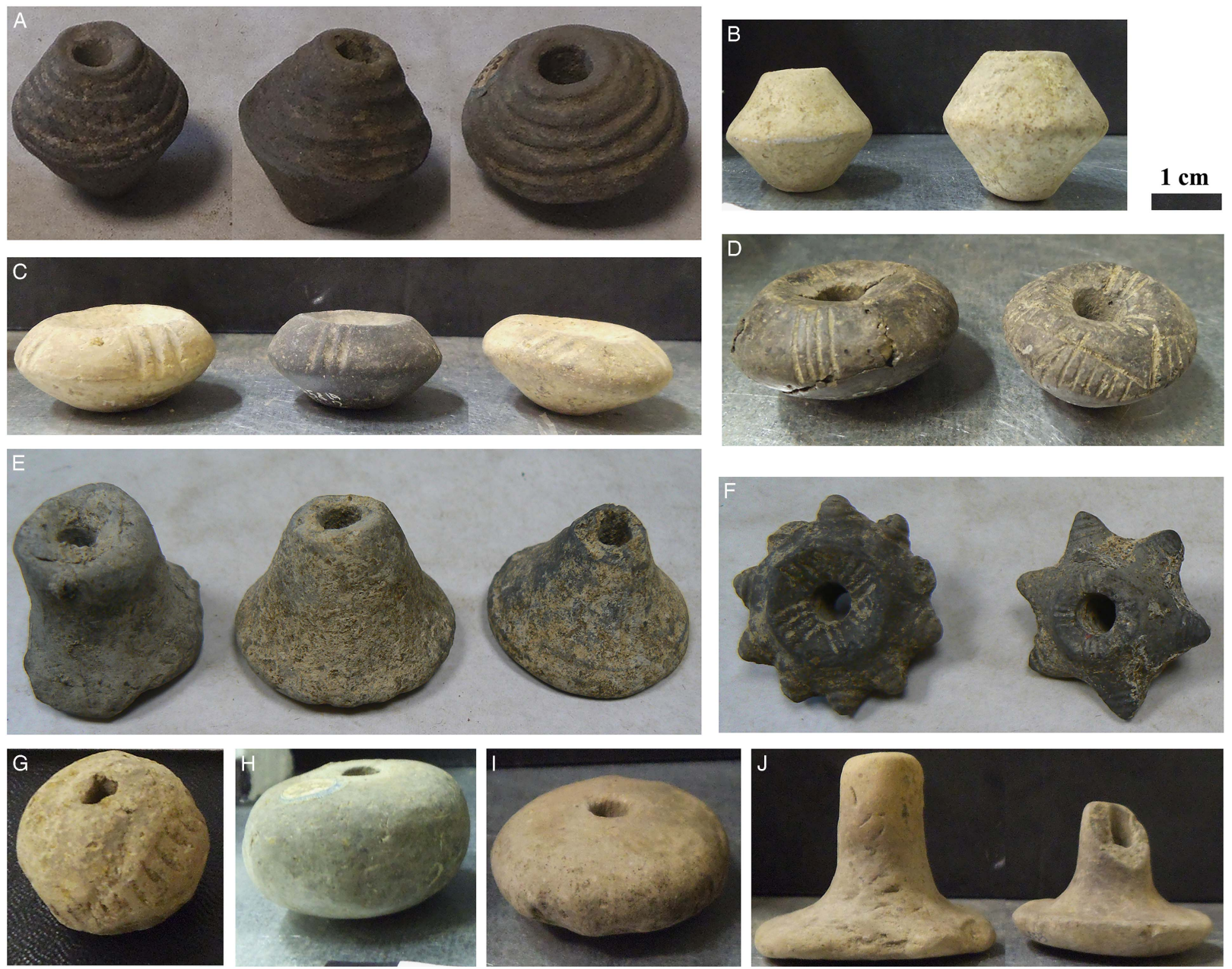

Fig. 4.

Examples of spindle whorls from Montale (photos: S. Sabatini)

primary export. Of course, spinning techniques could also have changed or clay spindle whorls might have been substituted by whorls made in other materials. ${ }^{9}$ Although definitive conclusions cannot be drawn, the consistent economic transformations that seem to have occurred at Montale at the beginning of the Recent Bronze Age are in chronological correspondence with the establishment of settlement hierarchies throughout the Terramare area (eg, Cardarelli 2015; Pacciarelli 2016, 168-72). Montale does not change its size with time, as other prominent Terramare settlements do, but interregional political dynamics probably played a major role in its particular economy.

To understand the social context of any specialised production, it is important to assess its possible link to the political economy that supported the emergence of social hierarchies (Earle \& Spriggs 2015). The role of specialisation in the formations of social hierarchies has been discussed archaeologically since at least Childe (1942). Brumfiel and Earle (1987b) have drawn attention to the ability to control some prestige goods, like cloth, by what they call attached specialisation, meaning simply that elites sponsored production and thus effectively controlled distribution of socially meaningful objects. Kristiansen (1987) has developed this argument for metal production in Scandinavia during the Bronze Age.

Although the characteristics of archaeological evidence from Montale makes it difficult to assess whether textile production in general, and spinning in 
TABLE 5: TYPES OF SPINDLE WHORLS FROM THE MODERN STRATIGRAPHIC EXCAVATIONS PER PHASE

\begin{tabular}{|c|c|c|c|c|c|c|c|c|c|c|c|}
\hline $\begin{array}{l}\text { Montale's phases } \\
\text { Whorls (stratigraphic excavations) } \\
\text { (fragmentary items) }\end{array}$ & & $\begin{array}{l}I \\
19 \\
(7) \\
\end{array}$ & $\begin{array}{c}I I \\
44 \\
(20) \\
\end{array}$ & $\begin{array}{l}I I I \\
31 \\
(15) \\
\end{array}$ & $\begin{array}{l}I V \\
17 \\
(11) \\
\end{array}$ & $\begin{array}{l}V \\
1 \\
(1) \\
\end{array}$ & $\begin{array}{l}V I \\
25 \\
(15) \\
\end{array}$ & $\begin{array}{l}\text { VII } \\
16 \\
(10) \\
\end{array}$ & $\begin{array}{l}\text { VIII } \\
12 \\
(8) \\
\end{array}$ & $\begin{array}{c}I X \\
16 \\
(10) \\
\end{array}$ & $\begin{array}{c}X \\
1 \\
(1) \\
\end{array}$ \\
\hline Type 1. Truncated conical & $1 \mathrm{a}$ & $3(1)$ & $2(0)$ & & & & $3(2)$ & $1(0)$ & & & \\
\hline (a) regular, & $1 \mathrm{~b}$ & & $3(0)$ & $1(0)$ & $2(2)$ & & & $1(1)$ & & & \\
\hline $\begin{array}{l}\text { (b) embossed profile, } \\
\text { (c) bell-shaped }\end{array}$ & $1 c$ & $1(1)$ & $1(1)$ & $1(1)$ & $1(1)$ & & & & $1(1)$ & $2(2)$ & \\
\hline Type 2. Biconical asymmetric & $2 \mathrm{a}$ & $10(2)$ & $12(3)$ & $3(0)$ & $1(1)$ & & $2(1)$ & $6(3)$ & $1(0)$ & $5(1)$ & \\
\hline (a) regular & $2 \mathrm{~b}$ & & $1(0)$ & $1(1)$ & & & & & & & \\
\hline $\begin{array}{l}\text { (b) flattened } \\
\text { (c) embossed profile }\end{array}$ & $2 c$ & $2(2)$ & $3(3)$ & $1(1)$ & & & & $2(1)$ & & $1(1)$ & \\
\hline $\begin{array}{l}\text { Type 3. Biconical asymmetric with } \\
\text { plastic protuberances }\end{array}$ & 3 & $3(1)$ & $3(1)$ & $1(1)$ & & & & & & & \\
\hline Type 4. Biconical & $4 \mathrm{a}$ & & $2(0)$ & $10(4)$ & $6(5)$ & $1(1)$ & $11(7)$ & & & $1(0)$ & \\
\hline $\begin{array}{l}\text { (a) regular, } \\
\text { (b) embossed profile, }\end{array}$ & $4 \mathrm{~b}$ & & $2(2)$ & $3(1)$ & $2(1)$ & & $3(2)$ & $1(1)$ & $7(5)$ & $3(3)$ & $1(1)$ \\
\hline Type 5. Biconical with concentric marks & $5 \mathrm{a}$ & & $5(2)$ & $5(2)$ & $2(0)$ & & $4(1)$ & $2(1)$ & & $1(0)$ & \\
\hline $\begin{array}{l}\text { (a) on one cone, } \\
\text { (b) on both cones, }\end{array}$ & $5 b$ & & $2(1)$ & $2(1)$ & & & & & & & \\
\hline Type 6. Convex-cylindrical & 6 & & $3(2)$ & & $1(0)$ & & & & & & \\
\hline Type 7. Disc-shaped & 7 & & $1(1)$ & & & & & & & & \\
\hline Type 8. Globular & 8 & & $1(1)$ & & $1(0)$ & & & & & $1(1)$ & \\
\hline Type 9. Pin-head & 9 & & & & & & & & $2(1)$ & & \\
\hline No type/too fragmentary & & & (3) & (3) & (1) & & (2) & (3) & (1) & (2) & \\
\hline
\end{tabular}




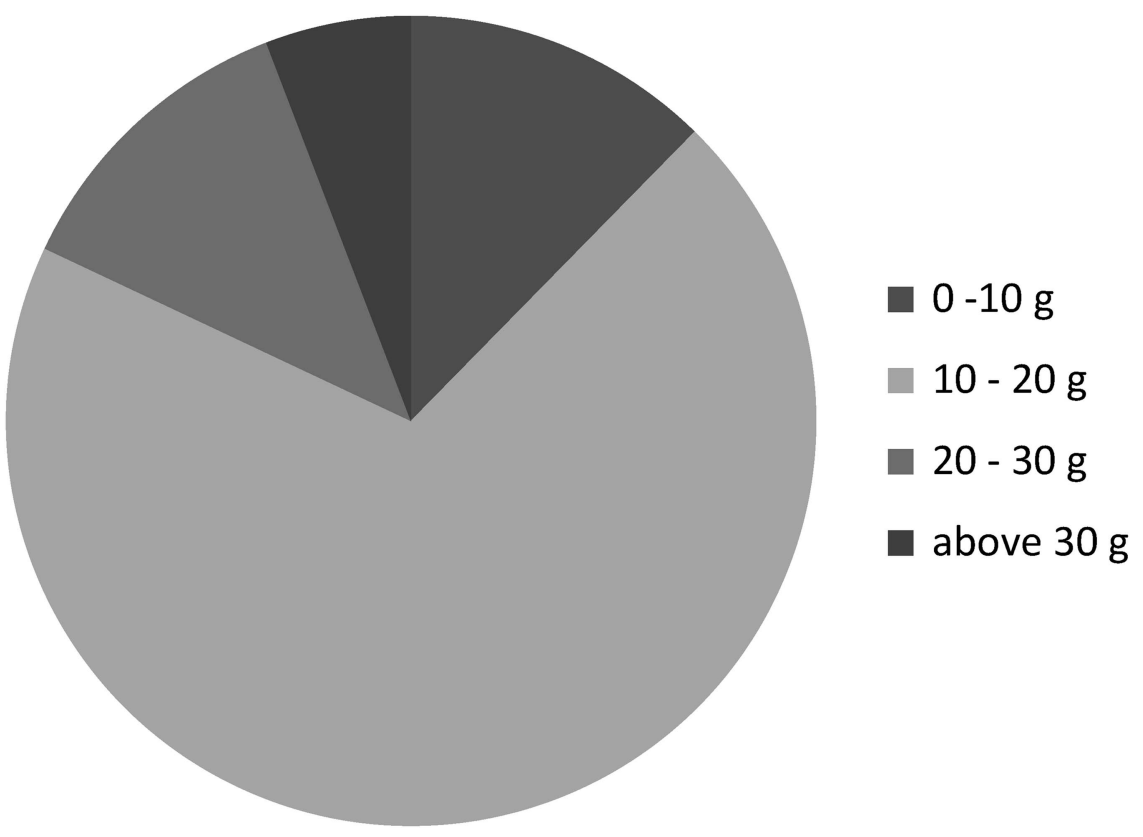

Fig. 5 .

Spindle whorls from the 19th century collection at Montale according to categories of weight $(\mathrm{N}=4089)$

particular, could have been controlled by an emerging Terramare elite, the timing of this specialised manufacture, which began much earlier than the emergence of settlement hierarchies in the Terramare area (at the end of the Middle Bronze Age 3), suggests that Bronze Age wool production, as documented by Montale, was not being channelled into manageable 'bottlenecks'. A bottleneck, such as attached specialisation or dominance of trade, is a point of restriction in the commodity chain, which would allow elites to channel the flow of critical commodities (Earle et al. 2015). More case studies are needed, but one could tentatively suggest that patterns of Terramare textile making and trade might have been rather deeply affected by external factors which determined profitability.

All in all, the huge quantity of spindle whorls recovered at Montale argues strongly for intensive yarn production. Additionally, considering the limited size of the settlement (c. 1 ha and a probable maximum population of $c$. 130 inhabitants, cf. Cardarelli 2015,167), a conspicuous part of the local population probably participated in such production. What can be said about the social context of this textile production, as evident at Montale? The lack of material culture signalling the existence of a welldefined elite is a recurrent issue in studies of the Terramare area attempting to assess modes of political and economic management and control (eg, Cardarelli 2015; Pacciarelli 2016). Our proposal, as far as textile specialisation at Montale is concerned, is to envisage a community-based (corporate) entrepreneurship. As mentioned, the existence of a corporate structure in Terramare settlements has also been proposed in order to explain the conspicuous and recurrent collective effort that must have been put into, among other things, digging and maintaining the large ditches and corresponding embankments normally surrounding local settlements (Cardarelli 2015, 168). A similar coordinated and communal effort has also been proposed for the construction of other features from the plain such as the imposing ritual basin of Noceto, Parma province, (Bernabò Brea \& Cremaschi 2009; Cremaschi \& Ferrari 2009, 106-7) or irrigation systems dated to the end of the Middle Bronze Age (Cardarelli 2015, 168). Even dismissing the hypothesis of attached production, it seems unlikely, given such premises, that single household or individual workshops actively pursued manufacturing activities for private wealth accumulation, as is suggested for independent, market-driven production models (Brumfiel \& Earle 1987b).

Rather, the high quantity of spindle whorls at Montale suggests a broad, community-based 


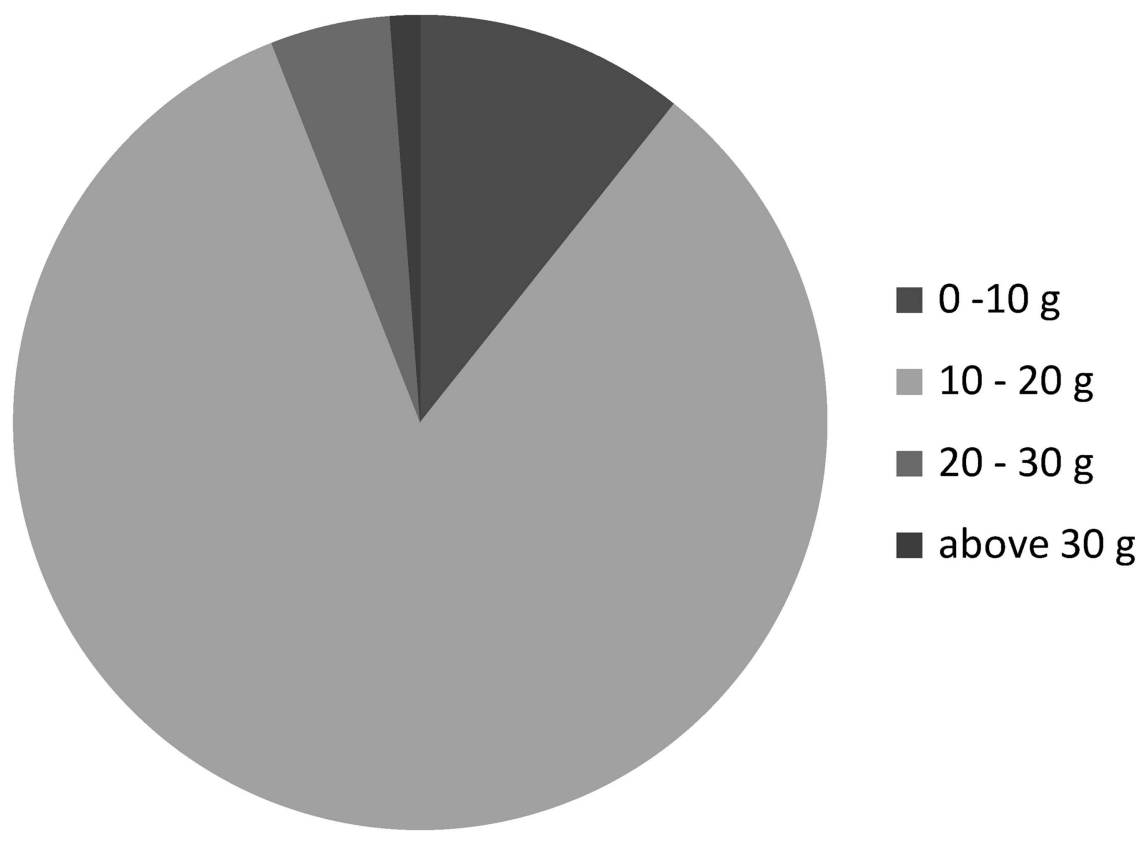

Fig. 6.

Spindle whorls from the stratigraphic excavations (1998-2002) at Montale according to categories of weight $(\mathrm{N}=84)$

specialisation, in which many spinners (or a large number of community members) participated. We propose that the characteristics of the archaeological record fit well with what has been called a 'community of practice'. Relying on an anthropological study of modern work groups (Wenger et al. 2002), communities of practice can be defined as close-knitted groups of specialists, often situated socially in single environments, communicating and sharing knowledge, exchanging services, training novices through social learning systems, and, at times applying practicebased social pressure to ensure that all participants conform and that expected outcomes are achieved (Wenger 2010; compare Santacreu et al. 2014). As to our concern with Terramare social organisation, in a community of practice, leadership and practice management ought to acquire an internal intrinsic legitimacy that enables the establishment of effective organisation (eg, Wenger 1998a; 1998b, 72-9). Such a community of practice can result in several characteristics, including concentrated specialist activities in one locale, standardised production methods, and a localised competitive advantage defined by 'the regime of competence' that social learning and shared knowledge create (cf. Wenger 2010). Certainly the concentration of spinning tools at
Montale easily fits the first criterion. The homogeneity of shapes within some of Montale's types of whorls (see Fig. 4), suggests also that the technology used in specialised textile production might have been produced in a standardised way (by the very same communities of practice or, perhaps, by workshops manufacturing these tools for the rest of the community?). Although further studies and analyses are necessary to test such hypotheses, the evidence hints at coordinated productive activities within the village. Looking specifically at weaving practices, a community of practice model has been also proposed to interpret the archaeological evidence for the Terramare loom weights (Sabatini in press). A comparative analyses of the available evidence from various sites of the Po plain and of the neighbouring Garda lake/Trentino area suggests that changes - in particular in the weight of the loom weights - occur contemporarily over the Po plain, but not in the Garda lake/Trentino area. In other words, weaving practices might not have been exclusive in the various villages, but rather shared widely. The known Terramare loom weight assemblages suggest that looms with unevenly heavy weights characterise Middle Bronze Age 2, while relatively light and similar weights generally 


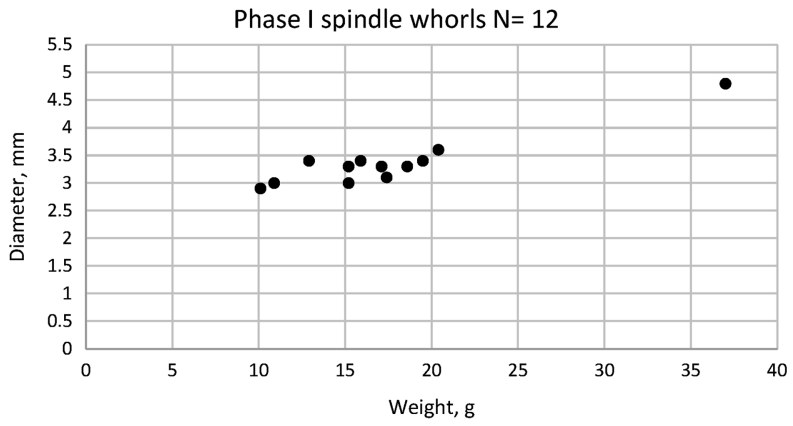

Phase III spindle whorls $\mathrm{N}=16$

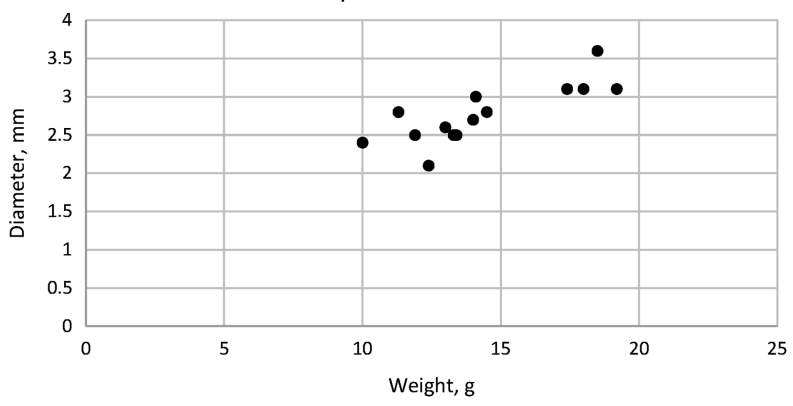

Phase VI spindel whorls $\mathrm{N}=10$

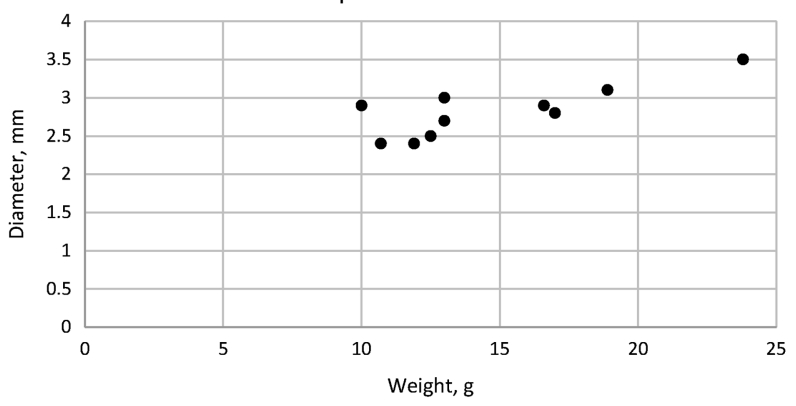

Phase VIII spindle whorls $\mathrm{N}=4$

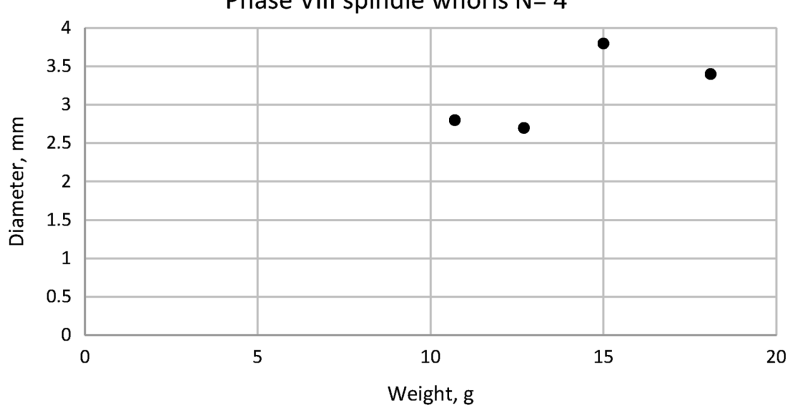

Phase II spindle whorls $\mathrm{N}=\mathbf{2 4}$

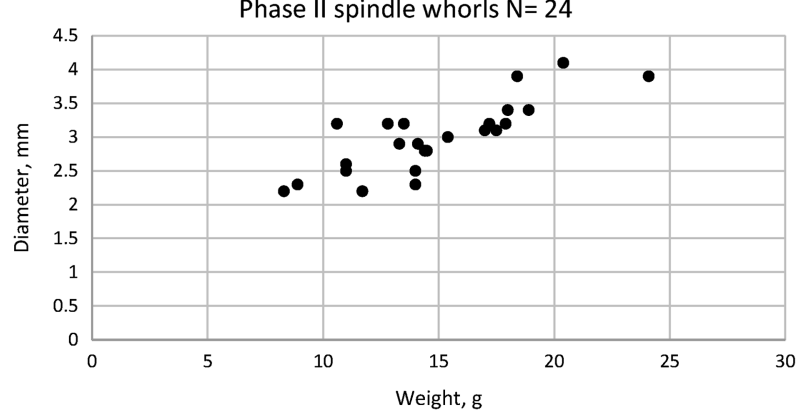

Phase IV spindle whorls $\mathrm{N}=6$

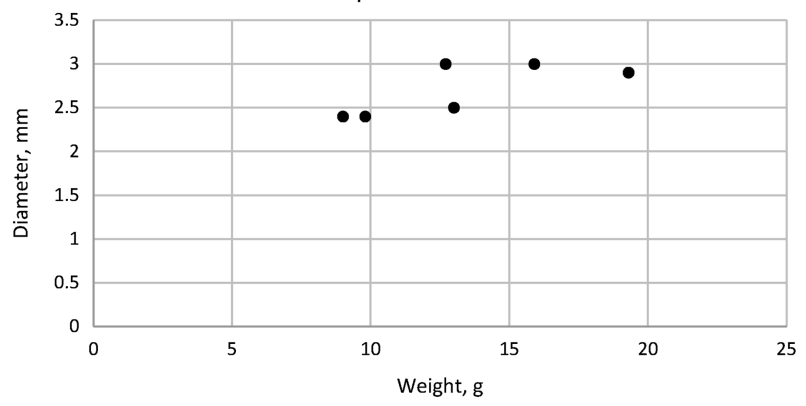

Phase VII spindle whorls $\mathrm{N}=6$
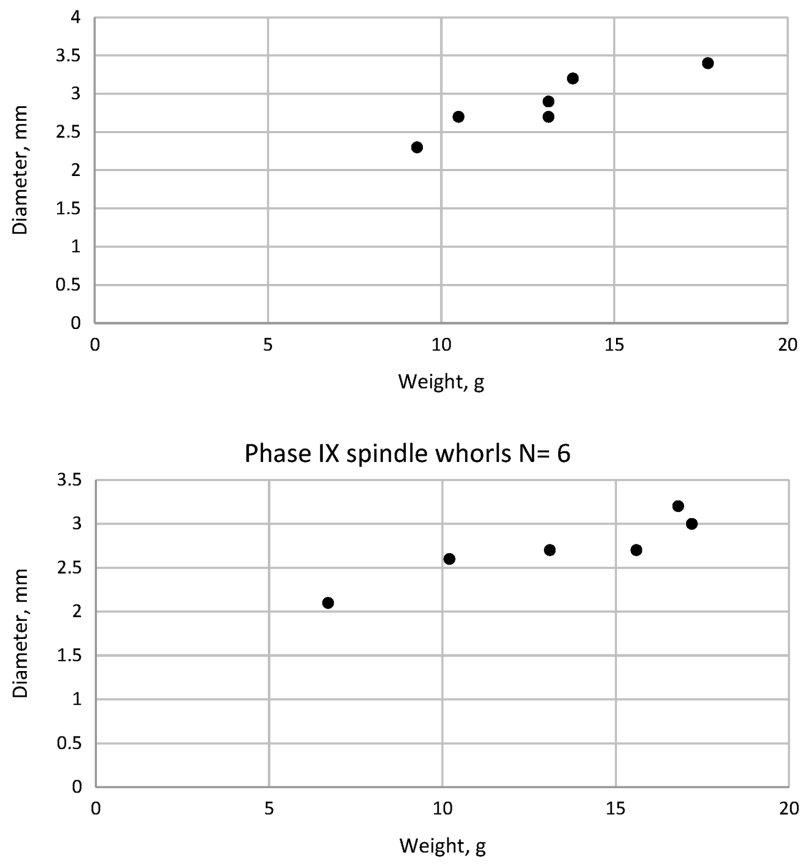

Fig. 7.

Scatter plot of the complete items from the stratigraphic excavations at Montale $(\mathrm{N}=84)$ by weight/diameter 
distinguished Middle Bronze Age 3 and Recent Bronze Age 1 (cf. Fig. 1) assemblages. During Recent Bronze Age 2 heavy items of 1-2 kg appear instead the most common loom weights. Although further research is necessary to provide a secure reconstruction of Bronze Age weaving traditions on the plain, it seemed reasonable to envisage Terramare weaving as a dynamic activity developed by communities of specialists who networked broadly across Terramare villages, actively negotiating and developing regional weaving technologies.

\section{CONCLUSIONS}

In addition to the well-known metal trade, textiles must have been important commodities in the Bronze Age generally (eg, Frei et al. 2015; 2017; Kristiansen 2016; Vandkilde et al. 2015). Textual evidence from the Aegean and the Near East documents how textile manufacture was closely supervised, involving the interplay of resource procurement and labour specialisation. The lack of written evidence and the poor archaeological preservation of textiles has limited prehistorians' ability to capture the organisation of Bronze Age textile economies outside the Mediterranean. Rather, outside this region, attention must focus on textile technologies and their tools, such as spindle whorls, which can be studied in terms of settlement and household variability as a means to describe labour specialisation and its multiple roles in the political economy (Sabatini in press). The evidence from Montale suggests that textile crafts could have consituted a major settlement specialisation based on the capacities of local population to exploit environmental and human resources, including specialised labour, and - as to wool production management of large herds. Montale's community might, therefore, have managed production to meet wider continental demands, as documented for northern Europe, where woollen clothing, at least during the 14th and the 13th centuries BCE, was used apparently without convincing signs for local wool production (Bergerbrant in press; Kristiansen \& Stig Sørensen in press).

But what was the political significance of specialised textile production at Montale? We propose a simple model that would help explain the pattern of textile specialisation in the broader context of what is known of the local communities' socio-political organisation
(Cardarelli 2009a; 2014; 2015). Terramare societies appear to have been oriented towards exploitation of their immediate territories with surplus productions in exchange for non-local commodities. Probably, already from Middle Bronze Age 3 (Fig. 1), a number of Terramare settlements employed irrigated agriculture (eg, Cremaschi et al. 2006, 89; Vanzetti 2013, 271-2), which must have involved fairly large-scale capital improvements. In order to defend their substantial investments and, most likely, the products of their labour, Terramare societies constructed their fortified settlements and imported metals for harvesting tools, weapons to defend their land, and elaborate ornaments and ritual objects (see Carancini 1997; Marzatico 1997) suggesting a hard-working but sophisticated society. To obtain unavailable raw materials such as metal, export products were essential. Montale, despite its limited size, has already attracted attention as applying a Thiessen polygon model indicates that it dominated an unusually large territory of 2200 ha, when compared with the average (260-890 ha) for the neighbouring sites of the province (Cardarelli 2009c, 43). Access to such a wide landscape might have allowed the Montale community to carry out both an intense agricultural production and animal husbandry at close range. In addition, Montale is situated in proximity to the southern margins of the plain, thus close to potential summer pastures offered by the Appenines which were abundantly used for sheep farming during Roman times, for example (Corti 2012). Combined with the archaeological evidence for intense sheep/goat husbandry (De Grossi Mazzorin \& Ruggini 2009), our hypothesis is that the favourable regional environmental conditions and the likely capacity for development of communities of practice in spinning and weaving created the necessary local comparative advantage that made surplus production and exports in wool feasible.

The evidence from Montale suggests that the role of specialised textile production in the European Bronze Age was highly variable and dependent on the broader political economy in which it was imbedded. This focus on variability is critical to understanding different forms of specialisation generally and the wool textile economy specifically. We encourage the investigation of textile production as a means by which to study alternative pathways for social formation, some emphasising hierarchy while others possibly remaining resistant to elite control. As it must 
be documented archaeologically, during the Bronze Age, communities and regions appear to have engaged in continent-wide trade in many commodities in quite different ways and with quite different effects.

Acknowledgements: Andrea Cardarelli kindly entrusted the archaeological material presented here to Serena Sabatini. His contribution provides the broad perspective on the Terrarmare culture. Access to the archaeological material was provided by the Civic Archaeological and Ethnographical Museum of Modena and generously assisted by Gianluca Pellacani, to whom goes our deep gratitude. Tim Earle, who invaluably contributed to the theoretical framing, was brought into the project, while a Visiting Scholar at Gothenburg University (May 2016). We are grateful to Helene Whittaker for reading and commenting on earlier drafts and to Kristian Kristiansen for engaging discussions and comments and to the anonymous reviewers.

This project was supported by the European Research Council under Advanced Researchers Grant no. 269442 Travels, Transmissions and Transformations in Temperate Northern Europe during the $3 r d$ and $2 n d$ Millennium $B C:$ The rise of Bronze Age societies; The Swedish Foundation for Humanities and Social Sciences under Project Grant P15-0591:1 Bronze Age Wool Economy: Production, trade, environment, herding and society; and Birgit och Gad Rausing Foundation under project grant Bronze Age Textiles in Southern Europe: Production, use, and exchange.

\section{Endnotes}

${ }^{1}$ The Bronze Age chronology for mainland Italy can be summarised as follows: Early Bronze Age (c. 2200-1700/ 1650 вс); Middle Bronze Age (c. 1700/1650-1325/1300 вС); Recent Bronze Age (c. 1325/1300-1150 BC); and Final Bronze Age (c. 1150-950/925 BC).

2 The excavations carried out at Castione dei Marchesi during the 19th century did not provide stratigraphic evidence and thus the possibility of securing material to a precise chronology. The site was in use between the Middle and the Recent Bronze Ages (cf. Fig. 1). Since the organic material came from the lowest levels, it is likely to have belonged to the Middle Bronze Age.

${ }^{3}$ The term terramara is, in local dialects, the name of the organic soil dug out in the 19th century from numerous local manure quarries. As evident even then, the quarries were the remains of Bronze Age settlements (eg, Bernabò Brea \& Mutti 1994; Saltini 1997). Available archaeological evidence today consists, therefore, of two main groups of material: the finds collected during the 19th century and those from modern excavations.

${ }^{4}$ Flax, hemp, nettle, and Tilia (lime bast) (all well-known taxa in textile production, see Barber 1991, 9-35) were present in the plain and possibly cultivated (Ravazzi et al. 2004; Mercuri et al. 2006; Aceti et al. 2009, 124; Rottoli \&
Castiglioni 2009). Thus, wool, was possibly not the only fibre manufactured.

${ }^{5}$ The hypothesis of warp weighted looms standing against the wall of some of Poviglio's structures has been formulated on the base of the distribution of loom weights archaeological evidence. Clear rows of loom weights have been found parallel to the walls in more than one structure (Bernabò Brea et al. 2003; Bianchi 2004).

${ }^{6}$ Montale's material has not yet been published in its entirety and there are no approximate numbers for all the implements named here, except for the spindle whorls and the loom weights; the latter have been thoroughly presented elsewhere (Sabatini in press).

${ }^{7}$ It is worth stressing that the high frequency of spindle whorls in the small area of the modern excavation and the limited number of loom weights, is in harmony with numbers and proportions of the 19th century collections confirming that, at Montale, major emphasis was probably put on yarn production.

${ }^{8}$ The phenomenon has not yet been scientifically addressed but existing publications of textile tools show a possibly similar trend at Poviglio during Recent Bronze Age 2 (Bianchi 2004, 611; Lincetto 2006, 119 \& 201).

${ }^{9} \mathrm{~A}$ case in point is represented by the wheel-like objects made of animal bone and horn which largely characterise Recent Bronze Age material culture from the plain. They have been interpreted as pin-heads, but also as possible spindle whorls (eg, Provenzano 1997, 533).

\section{BIBLIOGRAPHY}

Accorsi, C.A., Bandini Mazzanti, M., Bosi, G., Marchesini, M., Mercuri, A.M. \& Trevisan, G. 2009. The archaeobotanical analysis. In Cardarelli 2009b, 64-67

Aceti, A., Ravazzi, C. \& Vescovi, E. 2009. Analisi pollinica della successione stratigrafica. In Bernabò Brea \& Cremaschi 2009, 121-31

Andersson Strand, E. \& Cybulska, M. 2013.Visualising ancient textiles - how to make a textile visible on the basis of an interpretation of an ur III text. In M.L. Nosch, H. Koefoed \& E. Andersson Strand (eds), Textile Production and Consumption in the Ancient Near East, 113-27. Ancient Textiles Series 12. Oxford: Oxbow Books

Andersson Strand, E. \& Nosch, M.L. (eds). 2015a. Tools, Textiles and Contexts: Textile Production in the Aegean and Eastern Mediterranean Bronze Age. Ancient Textiles Series 21. Oxford: Oxbow Books

Andersson Strand, E. \& Nosch, M.L. 2015b. Introduction to the CTR database. In Andersson Strand \& Nosch 2015a, 144-51

Barber, E.J.W. 1991. Prehistoric Textiles: The development of cloth in the Neolithic and Bronze Ages with special references to the Aegean. Princeton: Princeton University Press

Bazzanella, M. 2012. Italy: Neolithic and Bronze Age. In Gleba \& Mannering 2012, 203-14 
Bazzanella, M. \& Mayr, A. 2009. I reperti tessili, le fusaiole e i pesi da telaio dalla palafitta di Molina di Ledro.Trento: Provincia Autonoma di Trento

Bender Jørgensen, L. 1992. North European Textiles until $A D$ 1000. Aarhus: Aarhus University Press

Bender Jørgensen, L. 2012. Spinning faith. In M.-L. Stig Sørensen \& K. Rebay-Salisbury (eds), Embodied Knowledge, 128-35. Oxford: Oxbow Books

Bender Jørgensen, L. \& Rast-Eicher, A. 2016. Innovation in European Bronze Age textiles. Praeistorische Zeitschrift 91(1), 68-102

Bergerbrant, S. 2007. Bronze Age Identities: Costume, conflict and contact in Northern Europe 1600-1300 BC. Lindome: Bricoleur Press

Bergerbrandt, S. in press. Local or traded? Wool textiles in the Early Nordic Bronze Age. In Sabatini \& Bergerbrant in press

Bernabò Brea, M. \& Cremaschi, M. (eds). 2004. Il villaggio piccolo della terramara di Santa Rosa di Poviglio. Scavi 1987-1992. Florence: Origines

Bernabò Brea, M. \& Cremaschi, M. (eds). 2009. Acqua e civiltà nelle Terramare, la vasca votiva di Noceto. Milan: Skira

Bernabò Brea, M. \& Mutti, A. 1994. '... Le terremare si scavano per concimare $i$ prati ...'. La nascita dell'archeologia preistorica a Parma nella seconda meta dell'Ottocento. Parma: Silva Editore

Bernabò Brea, M., Bianchi, P. \& Lincetto, S. 2003. La produzione tessile nell'età del Bronzo. Fusaiole e pesi da telaio nelle terramare emiliane: esempi di studio dai villaggi di S. Rosa di Poviglio (RE) e Forno del Gallo a Beneceto (PR). In M. Bazzanella, A. Mayr, L. Moser \& A. RastEicher (eds), Textiles. Intrecci e tessuti dalla preistoria europea, 111-20. Trento: Provincia Autonoma di Trento

Bernabò Brea, M., Cardarelli, A. \& Cremaschi, M. (eds). 1997a. Le Terramare. La più antica civiltà padana. Milan: Electa

Bernabò Brea, M., Cardarelli, A. \& Cremaschi, M. 1997b. L'insediamento collinare e montano. In Bernabò Brea et al. (eds) 1997a, 275-94

Bernabò Brea, M., Cremaschi, M. \& Pizzi, C. 2003. Le strutture abitative del Villaggio Grande -fase su palafittadella terramara di Santa Rosa di Poviglio (RE). In C. Peretto (ed.), Analisi informatizzata e trattamento dati delle strutture di abitato di età Preistorica $e$ Protostorica in Italia, 271-85. Florence: Origines

Blake, E. 2014. Social Networks and Regional Identity in Bronze Age Italy. Cambridge: Cambridge University Press

Bianchi, P. 2004. Manufatti per filatura e tessitura. In Bernabò Brea \& Cremaschi (eds) 2004, 609-51

Bianchi, P. \& Bernabò Brea, M. 2012. Rappresentazioni mobiliari zoomorfe, antropomorfe e simboliche dell'età del Bronzo. Preistoria alpina 46(2), 299-308

Biga, M.G. 2011. La lana nei testi degli Archivi Reali di Ebla (Siria, XXIV sec. a.C.): alcune osservazioni. In E. Ascalone $\&$ L. Peyronel (eds), Studi italiani di metrologia ed economia del vicino oriente antico dedicati a Nicola Parise in occasione del suo settantesimo compleanno, 77-92. Rome: Herder
Breniquet, C. \& Michel, C. 2014. Wool Economy in the Ancient Near East and the Aegean: From the beginnings of sheep husbandry to institutional textile industry. Ancient Textiles Series 17. Oxford: Oxbow Books

Broholm, H.C. \& Hald, M. 1940. Costumes of the Bronze Age in Denmark:Contributions to the archaeology and textile-history of the Bronze Age. Copenhagen: Arnold Busck

Brumfiel, E. \& Earle, T.K. (eds). 1987a. Specialization, Exchange, and Complex Societies. Cambridge: Cambridge University Press

Brumfiel, E. \& Earle, T.K. 1987b. Specialization, exchange, and complex societies: An introduction. In Brumfiel \& Earle (eds) 1987a, 1-9

Burke, B. 2010. Textiles. In E.H. Cline (ed.), The Oxford Handbook of the Aegean Bronze Age, 430-42. Oxford: Oxford University Press

Busana, M.S. \& Basso, P. (eds). 2012. La lana nella Cisalpina Romana. Padua: Padova University Press

Canci, A., Cupitò, M., Pulcini, M.L., Salzani, L., Fornaciari, G., Tafuri, M.A. \& Dalla Zuanna, G. 2015. La necropoli della media e recente età del bronzo di Olmo di Nogara (Verona). Risultati della ricerca osteoarcheologica. In G. Leonardi \& V. Tinè (eds), Preistoria e Protostoria del Veneto, 327-40. Florence: Istituto Italiano di Preistoria e Protostoria

Candelato, F., Cardarelli, A., Cattani, M., Labate, D. \& Pellacani, G. 2002. Il sistema informativo della scavo della terramara di Montale (Catselnuovo di Rangone-MO). In C. Peretto (ed.), Analisi informatizzata e trattamento dati delle strutture di abitato di età preistorica e protostorica in Italia, 257-70. Florence: Istituto Italiano di Preistoria e Protostoria

Carancini, G.L. 1997. La produzione metallurgica delle terramare nel quadro dell'Italia protostorica. In Bernabò Brea et al. 1997a, 379-404

Cardarelli, A. 2006. L'Appennino modenese nell'età del Bronzo. In A. Cardarelli \& L. Malnati (eds), Atlante dei beni archeologici della provincia di Modena, II, 40-68, Florence: All'Insegna del Giglio

Cardarelli, A. 2009a. The collapse of the terramare culture and growth of new economic and social systems during the Late Bronze Age in Italy. Scienze dell'antichità 15, 449-520

Cardarelli, A. (ed.) 2009b. Guide to the Archaeological Park and Open-Air Museum Terramara Montale. Modena: Museo Civico Archeologico Etnologico

Cardarelli, A. 2009c. Insediamenti dell'età del Bronzo fra Secchia e Reno. Formazione, affermazione e collasso delle terramare. In A. Cardarelli \& L. Malnati (eds), Atlante dei beni archeologici della provincia di Modena, III, 34-58, Florence: All'Insegna del Giglio

Cardarelli, A. (ed.) 2014. La necropoli della terramara di Casinalbo. Florence: All'Insegna del Giglio

Cardarelli, A. 2015. Different forms of social inequality in Bronze Age Italy. Origini 38 (2015-2), 151-200

Cardarelli, A. \& Labate, D. 2009a. Ditch and embankment. In Cardarelli 2009b, 28-31 
Cardarelli, A. \& Labate, D. 2009b. The excavations 19962001. In Cardarelli (ed.) 2009b, 32-51

Cardarelli, A., Bosi, G., Rinaldi, R. Ucchesu, U. \& Bacchetta, G. Forthcoming. Vino o non vino? In Preistoria del cibo. L'alimentazione nella preistoria e nella protostoria (Atti della L Riunione Scientifica IIPP). Florence: Istituto Italiano di Preistoria e Protostoria

Cavazzuti, C. \& Putzolo, C. 2015. Strategie di occupazione dell'appennino emiliano durante l'età del bronzo. In F. Cambi, G. De Venuto \& R. Goffredo (eds), I PASCOLI, I CAMPI, IL MARE Paesaggi d'altura e di pianura in Italia dall'Età del Bronzo al Medioevo, 51-71. Bari: Edipuglia

CinBa database. Database of Bronze Age textiles in Europe. http://cinba.net/outputs/databases/textiles/ (last accessed 30 April 2018)

Childe, V.G. 1942. What Happened in History? New York: Harmondsworth

Corti, C. 2012. L'economia della lana a Mutina. In Busana \& Basso (eds) 2012, 213-29

Costin, C.L. 1991. Craft specialization: Issues in defining, documenting, and exploring the organization of production. Archaeological Method and Theory 3, 1-56

Cremaschi, M. \& Ferrari, P. 2009. Struttura e tecniche di costruzione della vasca. In Bernabò Brea \& Cremaschi (eds) 2009, 104-11

Cremaschi, M., Pizzi, C. \& Valsecchi, V. 2006. Management and land use in the terramare and a possible climatic cofactor in their abandonment: the case study of the terramara of Poviglio Santa Rosa (northern Italy). Quaternary International 151, 87-98

De Grossi Mazzorin, J. 2013. Considerazioni sullo sfruttamento animale in ambito terramaricolo. In J. De Grossi Mazzorin, A. Curci \& G. Giacobini (eds), Economia $e$ ambiente nell'Italia padana dell'età del bronzo. Le indagini bioarcheologiche, 257-63. Bari: Edipuglia

De Grossi Mazzorin, J. \& Ruggini, C. 2009. The archaeozoological analysis. In Cardarelli (ed.) 2009b, 68-9

Del Freo, M., Nosch, M.L. \& Rougemont, F. 2010. The terminology of textiles in the Linear B tablets, including some considerations on Linear A logograms and abbreviations. In Michel \& Nosch 2010, 338-73

Desantis, P. 2011. Aspetti simbolico religiosi. In P. Desantis, M. Marchesini \& S. Marvelli (eds), Anzola al tempo delle Terramare, 38-9. San Giovanni in Persiceto: Museo Archaeologico Ambientale

Earle, T. 2017. Property in prehistory. In Graziadei, M. \& L. Smith (eds), Comparative Property Law: Global perspectives, 3-25. Northampton, MA: Elgar

Earle, T. \& Spriggs, M. 2015. Political economy in prehistory: A Marxist approach to Pacific sequences. Current Anthropology 56(4), 515-44

Earle, T., Ling, J., Uhnér, C., Stos-Gale, Z. \& Melheim, L. 2015. The political economy and metal trade in Bronze Age Europe: Understanding regional variability in terms of comparative advantage and articulations. European Journal of Archaeology 18(4), 633-57

Firth, R. 2014. Considering the population statistics of the sheep listed in the east-west corridor archive at Knossos.
In D. Nakassis, J. Gulizio \& S.A. James (eds), KE-RAME-JA Studies Presented to Cynthia W. Shelmerdine, 293-304. Philadelphia: INSTAP

Firth, R. \& Nosch, M.L. 2012. Spinning and weaving wool in Ur III administrative texts. Journal of Cuneiform Studies 64, 67-84.

Frei, K.M., Mannering, U., Vanden Berghe, I. \& Kristiansen, K. 2017. Bronze Age wool: provenance and dye investigations of Danish textiles. Antiquity 91(357), 640-54

Frei, K.M., Mannering, U., Kristiansen, K., Allentoft, M.E., Wilson, A.S.,Tridico, S., Nosch, M.L., Willerslev, E., Clarke, L. \& Frei, R. 2015. Tracing the dynamic life story of a Bronze Age Female. Scientific Reports 5 (art. n. 10431; https://www.ncbi.nlm.nih.gov/pubmed/25994525

Gillis, C. \& Nosch, M.L. (eds). 2007. Ancient Textiles, Production, Craft and Society. Ancient Textiles Series 1. Oxford: Oxbow Books

Gleba, M. 2008. Textile Production in Pre-Roman Italy. Ancient Textiles Series 4. Oxford: Oxbow Books

Gleba, M. 2012. From textiles to sheep: investigating wool fibre development in pre-Roman Italy using scanning electron microscopy (SEM). Journal of archaeological Science 39, 3643-61

Gleba, M. \& Mannering, U. (eds). 2012. Textiles and Textile Production in Europe from Prehistory to AD 400. Ancient Textiles Series 11. Oxford: Oxbow Books

Grömer, K. 2013. Discovering the people behind the textiles: Iron Age textile producers and their products in Austria. In M. Gleba \& J. Pásztókai-Szeöke (eds), Making Textiles in Pre-Roman and Roman Times. People Places, Identities, 30-59. Ancient Textiles Series 13. Oxford: Oxbow Books

Grömer, K. 2016 The Art of Prehistoric Textile Making The Development of Craft Traditions and Clothing in Central Europe. Vienna: Verlag des Naturhistorischen Museums

Grömer, K., Kern, A., Reschreiter, H. \& RöselMautendorfer, H. 2013. Textiles from Hallstatt Weaving Culture in Bronze Age and Iron Age Salt Mines. Budapest: Archaeolingua

Guzowska, M., Becks, R., Andersson Strand, E., Cutler, J. \& Nosch, M.L. 2015. Textile tools from Troia, western Anatolia. In Andersson Strand \& Nosch 2015a, 309-28

Halstead, P. 1999. Texts, bones and herders: approaches to animal husbandry in Late Bronze Age Greece. Minos 33-4, 149-89

Harlow, S., Michel, C. \& Nosch, M.L. (eds). 2014. Prehistoric, Ancient Near Eastern and Aegean Textiles and Dress. Ancient Textiles Series 18. Oxford: Oxbow Books

Kania, K. 2013. Soft yarns, hard facts? Evaluating the results of a large-scale hand-spinning experiment. Archaeological and Anthropological Sciences 7(1), 113-30

Killen, J.T. 2007. Cloth production in Late Bronze Age Greece: the documentary evidence. In Gillis \& Nosch (eds) 2007, 50-9

Killen, J.T. 2015. Economy and administration in Mycenaean Greece. Collected Papers on Linear B Vol. 1 (ed. M. Del Freo). Rome: Incunabula Graeca CIV 
Kneisel, J. \& Schaefer, S. in press. Loom weights in Bronze Age Central Europe: Unravelling change in textile production. In Sabatini \& Bergerbrant in press

Kristiansen, K. 1987. From stone to bronze: the evolution of social complexity in northern Europe, 2300-1200 BC. In Brumfield \& Earle 1987a, 30-51

Kristiansen, K. 2016. Interpreting Bronze Age trade and migration. In E. Kiriatzi \& C. Knappet (eds) Human Mobility and Technological Transfer in the Prehistoric Mediterranean, 128-53. Cambridge: Cambridge University Press

Kristiansen, K. \& Stig Sørensen, M.-L. in press. Wool in the Bronze Age. Or: the Wool Age. In Sabatini \& Bergerbrant in press

Leonardi, G. 2012. Fusaiole 'in forma di vaso' e produzioni femminili nella protostoria: un problema aperto. In Busana \& Basso 2012, 339-51

Lincetto, S. 2006. Attività di filature e tessitura negli abitati e nelle abitazioni dell'età del bronzo dell'Italia settentrionale. Unpublished $\mathrm{PhD}$ thesis, Rome, University La Sapienza

Liu, R.K. 1978. Spindle whorls: Pt 1. Bead Journal 3, 87-103

Marzatico, F. 1997. La produzione metallurgica nelle terramare:sviluppo dei tipi e delle tecniche. In Bernabò Brea et al. 1997a, 577-86

McCorriston, J. 1997. The fiber revolution. Textile extensification, alienation and social stratification in Ancient Mesopotamia. Current Anthropology 38(4), 517-49

Mercuri, A.M., Accorsi, C.A., Bandini Mazzanti, M., Bosi, G., Cardarelli, A., Labate, D., Marchesini, M. \& Trevisan, G. 2006. Economy and environment of Bronze Age settlements - Terramaras - on the Po Plain (northern Italy): first results from the archaeobotanical research at the Terramara di Montale. Vegetation History \& Archaeobotany 16, 43-60

Michel, C. 2014. Wool trade in Upper Mesopotamia and Syria according to Old Babylonian and Old Assyrian texts. In Breniquet \& Michel 2014, 232-54

Michel, C. \& Nosch, M.L. (eds). 2010. Textile Terminologies in the Ancient Near East and Mediterranean from the Third to the First Millennia BC. Ancient Textiles Series 8. Oxford: Oxbow Books

Michel, C. \& Veenhof, K.R. 2010. The textile traded by the Assyrians in Anatolia (19th-18th centuries BC). In Michel \& Nosch (eds) 2010, 210-71

Nosch, M.L. 2011. The Mycenaean administration of textile production in the Palace of Knossos: Observations on the Lc(1) textile targets. American Journal of Archaeology 115(4), 495-505

Nosch, M.L. 2015. The Wool Age: Traditions and innovations in textile production, consumption and administration in the Late Bronze Age Aegean. In J. Weilhartner \& F. Ruppenstein (eds), Tradition and Innovation in the Mycenaean Palatial Polities, 167-201. Vienna: Austrian Academy of Science Press

Olofsson, L., Andersson Strand, E. \& Nosch, M.L. 2015. Experimental testing of Bronze Age textile tools. In Andersson Strand \& Nosch 2015, 75-100
Pacciarelli, M. 2016. The earliest processes towards citystates, political power and social stratification in the middle tyrrhenian Italy. Origini 39(1), 165-202

Provenzano, N. 1997. Produzione in osso e corno delle terramare emiliane. In Bernabò Brea et al. (eds) 1997a, 524-44

Pulcini, M.L. 2014. La necropoli di Olmo di Nogara (Verona). Studio paleobiologico dei resti umani per la ricostruzione dell'organizzazione di una comunità dell'Età del bronzo padana. Unpublished $\mathrm{PhD}$ thesis, University of Padua

Pulini, I. \& Righi, E. 2009. Plaiting, textiles and clothing. In Cardarelli (ed.) 2009b, 100-5

Randsborg, K. 2011. Bronze Age Textiles: Men, women and wealth. London: Bristol Classical Press

Rast-Eicher, A. \& Bender Jørgensen, L. 2013. Sheep wool in Bronze Age and Iron Age Europe. Journal of Archaeological Science 40(2), 1224-41

Ravazzi, C., Cremaschi, M. \& Forlani, L. 2004. Studio archeopalinologico. Nuovi dati, analisi floristica e sintassonomica della vegetazione dell'età del Bronzo. In Bernabò Brea \& Cremaschi 2004, 703-36

Riedel, A. 1989. L'economia animale (della terramara di Poviglio). In M. Bernabò Brea \& M. Cremaschi (eds), La Terramara di Poviglio. Le campagne di scavo 1985-1989, 37-8. Reggio Emilia: Origines

Riedel, A. 2004. La fauna. In M. Bernabò Brea \& M. Cremaschi (eds), Il Villaggio Piccolo della terramara di S. Rosa di Poviglio (Scavi 1987/1992), 703-36. Florence: Istituto Italiano di preistoria e protostoria

Robson, D. \& Ekarius, C. 2011. The Fleece \& Fiber Sourcebook. North Adams, MA: Storey

Rottoli, M. \& Castiglioni, E. 2009. Indagini sui resti macroscopici. In Bernabò Brea \& Cremaschi 2009, 152-63

Sabatini, S. in press. Weaving at the Bronze Age terramare settlement of Montale, northern Italy. In Sabatini \& Bergerbrant in press

Sabatini, S. In press. Wool economy during the European Bronze Age. Swiatowit, Annual of the Institute of Archaeology of the University of Warsaw 56

Sabatini, S. \& Bergerbrant, S. in press. The Textile Revolution in Bronze Age Europe. Cambridge: Cambridge University Press

Saltini, A. 1997. L'estrazione della 'terra mara' un'industria rurale nell'Emilia dell'Ottocento. In Bernabò Brea et al. (eds), Le Terramare. La più antica civiltà padana, 87-195. Milan: Electa

Santacreu, D.A., Vidal, A., García, J. and Calvo, M. 2014. Communities of practice and potter's experience: A case study from Southwestern Mallorca (c. 500-50 BC). Unpublished paper presented in 'Artisans Rule: Product standardization and craft specialization in prehistoric society' at 20th Annual Meeting of European Association of Archaeologists, Istanbul

Siennika, M. 2014. Changes in textile production in Late Bronze Age Tiryns, Greece. In K. Droß-Krüpe (ed.), Textile Trade and Distribution in Antiquity [Textilhandel und -distribution in der Antike], 161-76. Wiesbaden: Harrassowitz 
Skals, I., Möller-Wiering, S. \& Nosch, M.L. 2015. Survey of archaeological textile remains from the Aegean and Eastern Mediterranean area. In Andersson Strand \& Nosch 2015a, 61-74

Sofaer, J., Bender Jørgensen, L. \& Choyke, A. 2013. Craft production: ceramics, textiles and bone. In A. Harding \& H. Fokkens (eds), The Oxford Handbook of European Bronze Age, 469-91. Oxford: Oxford University Press

Vandkilde, H., Hansen, S., Kotsakis, K., Kristiansen, K., Müller, J., Sofaer, J. \& Stig Sørensen, M.L. 2015. In P. Suchowska-Ducke, S. Scott Reiter \& H. Vandkilde (eds), Cultural Mobility in Bronze Age Europe, in Forging Identities. The Mobility of Culture in Bronze Age Europe, 5-37. Oxford British Archaeological Report S2771

Vanzetti, A. 2013. 1600? The rise of the Terramara system (northern Italy). Tagungen des Landesmuseums für Vorgeschichte Halle 9, 267-82

Vretemark, M. 2010. Subsistence strategies. In T. Earle \& K. Kristiansen (eds), Organizing Bronze Age Societies, 155-84. Cambridge: Cambridge University Press
Waetzoldt, H. 1972. Untersuchungen zur neusumerischen Textilindustrie. Rome: Centro per le antichità e la storia dell'arte del Vicino Oriente

Wenger, E. 1998a. Communities of Practice: Learning, meaning, and identity. Cambridge: Cambridge University Press

Wenger, E. 1998b. Communities of practice: Learning as a social system. The Systems Thinkers 9(5). https:// thesystemsthinker.com/communities-of-practice-learningas-a-social-system/

Wenger, E. 2010. Communities of practice and social learning systems: the career of a concept. In C. Blackmore (ed.), Social Learning Systems and Communities of Practice, 179-98. New York: Springer and the Open University

Wenger, E., McDermott, R. \& Snyder, W. 2002. Cultivating Communities of Practice: A guide to managing knowledge. Cambridge: Harvard Business School Press

Wisti Lassen, A. 2010. The trade on wool in old Assyrian Anatolia. Jaarbericht Ex Oriente Lux 42, 159-79

Wright, R.P. 2013. Sumerian and Akkadian industries: crafting textiles. In H. Crawford (ed.), The Sumerian World, 395-418. London: Routledge 


\section{RÉSUMÉ}

Economie de la laine et du textile de l'âge du Bronze: Le cas du site de Terramare à Montale, Italie, de Serena Sabatini, Timothy Earle, et Andrea Cardarelli

A l'aube du 2ème millénaire avant J.-C. une économie lainière a fait surface à travers l'Europe continentale . Des sources archéologiques, iconographiques et écrites provenant du Proche Orient et de l'Egée attestent que l'économie de la laine à l'âge du bronze impliquait un considérable travail spécialisé et un élevage d'animaux à grande échelle. Ne reposant que sur des témoignages archéologiques, la connaissance détaillée des économies lainières dans l'Europe de l'âge du bronze était limitée, mais de récentes investigations du site de Montale à Terramare dans le nord de l'Italie documentent une densité élevée de fusaïoles et de poids qui confirme avec force l'existence de fabrication spécialisée de fil au niveau du village. La production ne semble pas avoir été rattachée à l'émergence d'une élite et n'était pas non plus totalement indépendante de contraintes sociales. Nous proposons que, bien que probablement dirigée par des élites locales, ,la production de laine était une entreprise basée sur la communauté et orientée vers l'exportation afin d'obtenir des matières premières et des biens qui n'étaient pas disponibles localement

\section{ZUSSAMENFASSUNG}

Textil- und Wollwirtschaft der Bronzezeit: Das Beispiel der Terramare-Siedlung von Montale, Italien, von Serena Sabatini, Timothy Earle, und Andrea Cardarelli

$\mathrm{Zu}$ Beginn des 2. Jahrtausends BC entwickelte sich eine Wollwirtschaft im gesamten kontinentalen Europa. Archäologische, ikonographische und schriftliche Quellen aus dem Vorderen Orient und der Ägäis zeigen, dass die bronzezeitliche Wollwirtschaft eine beträchtliche spezialisierte Arbeit sowie Viehzucht in großem Maßstab umfasste. Bezog sich die Forschung allein auf archäologische Daten, blieben die Erkenntnisse zur Wollwirtschaft im Europa der Bronzezeit beschränkt, aber jüngere Untersuchungen in der Terramare-Siedlung von Montale in Norditalien belegen eine große Dichte von Spinnwirteln, die deutlich für die Existenz von spezialisierter Manufaktur von Garn auf der Ebene von Dörfern spricht. Die Produktion scheint nicht mit dem Entstehen einer Elite zusammenzuhängen, noch war sie gänzlich unabhängig von sozialen Einschränkungen. Wir schlagen vor, dass die Herstellung von Wolle, auch wenn sie wahrscheinlich von lokalen Eliten geleitet wurde, eine gemeinschaftsbasierte Unternehmung war, ausgerichtet auf den Export mit dem Ziel, vor Ort nicht erhältliche Rohmaterialien und Güter zu erlangen.

\section{RESUMEN}

La economía de los textiles y de la lana en la Edad del Bronce: el caso del yacimiento de Terramare, Italia, por Serena Sabatini, Timothy Earle, y Andrea Cardarelli

En el inicio del II milenio cal вC surge la economía de la lana a lo largo del continente europeo. Las fuentes arqueológicas, iconográficas y escritas procedentes de Oriente Medio y del Egeo muestran que la economía de la lana en la Edad del Bronce supuso un considerable trabajo especializado y la cría de animales a gran escala. Centrándonos sólo en la evidencia arqueológica, el conocimiento detallado de las economías de la lana de la Edad del Bronce ha sido limitado, pero recientes investigaciones en el yacimiento de Terramare de Montale, en el norte de Italia, han documentado una elevada densidad de fusayolas que permiten sostener la existencia de una producción especializada de hilo a escala de aldea. Esta producción no parece haber estado vinculada a una élite emergente ni era completamente independiente de las restricciones sociales. Proponemos que, aunque probablemente estuvo gestionada por élites locales, la producción de lana fue un esfuerzo comunitario orientado a la exportación con el objetivo de obtener materias primas y bienes no disponibles en el entorno local. 